\title{
Weighted Differentiation Composition Operator from Logarithmic Bloch Spaces to Zygmund-Type Spaces
}

\author{
Huiying Qu, Yongmin Liu, and Shulei Cheng \\ School of Mathematics and Statistics, Jiangsu Normal University, Xuzhou 221116, China \\ Correspondence should be addressed to Yongmin Liu; minliu@jsnu.edu.cn
}

Received 7 January 2014; Accepted 10 March 2014; Published 17 April 2014

Academic Editor: Giovanni P. Galdi

Copyright (C) 2014 Huiying Qu et al. This is an open access article distributed under the Creative Commons Attribution License, which permits unrestricted use, distribution, and reproduction in any medium, provided the original work is properly cited.

Let $H(\mathbb{D})$ denote the space of all holomorphic functions on the unit disk $\mathbb{D}$ of $\mathbb{C}, u \in H(\mathbb{D})$ and let $n$ be a positive integer, $\varphi$ a holomorphic self-map of $\mathbb{D}$, and $\mu$ a weight. In this paper, we investigate the boundedness and compactness of a weighted differentiation composition operator $\mathscr{D}_{\varphi, u}^{n} f(z)=u(z) f^{(n)}(\varphi(z)), f \in H(\mathbb{D})$, from the logarithmic Bloch spaces to the Zygmundtype spaces.

\section{Introduction}

Let $\mathbb{D}$ denote the open unit disk of the complex plane $\mathbb{C}$ and $H(\mathbb{D})$ the space of all analytic functions in $\mathbb{D}$.

The logarithmic Bloch space is defined as follows:

$$
\begin{aligned}
\mathscr{B}_{\log }= & \{f \in H(\mathbb{D}): \\
& \left.\|f\|=\sup _{z \in \mathbb{D}}\left(1-|z|^{2}\right)\left(\log \frac{2}{1-|z|}\right)\left|f^{\prime}(z)\right|<\infty\right\} .
\end{aligned}
$$

The space $\mathscr{B}_{\log }$ is a Banach space under the norm $\|f\|_{\mathscr{B}_{\log }}=$ $|f(0)|+\|f\|$. Let $\mathscr{B}_{\log , 0}$ denote the subspace of $\mathscr{B}_{\log }$ consisting of those $f \in \mathscr{B}_{\log }$ such that

$$
\lim _{|z| \rightarrow 1}\left(1-|z|^{2}\right)\left(\log \frac{2}{1-|z|}\right)\left|f^{\prime}(z)\right|=0
$$

It is obvious that there are unbounded $\mathscr{B}_{\log }$ functions. For example, consider the function $f(z)=\log \log (e /(1-z))$. There are also bounded functions that do not belong to $\mathscr{B}_{\log }$. In fact, the interpolating Blaschke products do not belong to $\mathscr{B}_{\log }$. It is easily proved that, for $0<\alpha<$ $1, \mathscr{B}^{\alpha} \varsubsetneqq \mathscr{B}_{\log } \varsubsetneqq \mathscr{B} . \mathscr{B}_{\log }$ first appeared in the study of boundedness of the Hankel operators on the Bergman space. Attele in [1] proved that, for $f \in L_{a}^{2}(\mathscr{D})$, the Hankel operator $H_{f}: L_{a}^{1}(\mathscr{D}) \rightarrow L^{1}(\mathscr{D})$ is bounded if and only if $\|f\|_{\mathscr{B}_{\log }}<\infty$, thus giving one reason, and not the only reason, why log-Bloch-type spaces are of interest. Ye in [2] proved that $\mathscr{B}_{\log , 0}$ is a closed subspace of $\mathscr{B}_{\log }$. Galanopoulos in [3] characterized the boundedness and compactness of the composition operator $C_{\varphi}: \mathscr{B}_{\log } \rightarrow Q_{\log }^{p}$ and the boundedness and compactness of the weighted composition operator $u C_{\varphi}: \mathscr{B}_{\log } \rightarrow \mathscr{B}_{\log }$. Ye in [4] characterized the boundedness and compactness of the weighted composition operator $u C_{\varphi}$ between the logarithmic Bloch space $\mathscr{B}_{\log }$ and the $\alpha$-Bloch spaces $\mathscr{B}^{\alpha}$ on the unit disk and the boundedness and compactness of the weighted composition operator $u C_{\varphi}$ between the little logarithmic Bloch space $\mathscr{B}_{\log }^{0}$ and the little $\alpha$-Bloch spaces $\mathscr{B}_{0}^{\alpha}$ on the unit disk. Li in [5] characterized the boundedness and compactness of the weighted composition operator $u C_{\varphi}$ from Bergman spaces $A_{\beta}^{p}$ into the logarithmic Bloch space $\mathscr{B}_{\log }$ on the unit disk. Ye in [6] characterized the boundedness and compactness of the weighted composition operator $u C_{\varphi}$ from the general function space $F(p, q, s)$ into the logarithmic Bloch space $\mathscr{B}_{\log }$ on the unit disk. Colonna and $\mathrm{Li}$ in [7] studied the boundedness and compactness of the weighted composition operators from Hardy space into the logarithmic Bloch space and the little logarithmic Bloch space. Petrov in [8] obtains sharp reverse estimates for the 
logarithmic Bloch spaces on the unit disk. Castillo et al. in [9] characterized the boundedness and compactness of the composition operator from the logarithmic Bloch spaces into weighted Bloch spaces. García Ortiz and Ramos-Fernández in [10] characterized the boundedness and compactness of the composition operators from logarithmic Bloch spaces into Bloch-type spaces.

Let $\mu$ be a weight; that is, $\mu$ is a positive continuous function on $\mathbb{D}$. The Zygmund-type space $\mathscr{Z}_{\mu}$ consists of all $f \in H(\mathbb{D})$ such that

$$
\sup _{z \in \mathbb{D}} \mu(z)\left|f^{\prime \prime}(z)\right|<\infty .
$$

With the norm $\|f\|_{\mathscr{E}_{\mu}}=|f(0)|+\left|f^{\prime}(0)\right|+\sup _{z \in \mathbb{D}} \mu(z)\left|f^{\prime \prime}(z)\right|$, it becomes a Banach space. The little Zygmund-type space $\mathscr{Z}_{\mu, 0}$ is a subspace of $\mathscr{Z}_{\mu}$ consisting of those $f \in \mathscr{Z}_{\mu}$ such that

$$
\lim _{|z| \rightarrow 1} \mu(z)\left|f^{\prime \prime}(z)\right|=0
$$

When $\mu(z)=1-|z|^{2}$, the Zygmund-type space $\mathscr{Z}_{\mu}$ becomes the Zygmund space $\mathscr{Z}$ [11], while the little Zygmund-type space $\mathscr{Z}_{\mu, 0}$ becomes the little Zygmund space $\mathscr{Z}_{0}$.

Let $\mathscr{D}=\mathscr{D}^{1}$ be the differentiation operator; that is, $\mathscr{D} f=f^{\prime}$. If $n \in \mathbb{N}_{0}$, then the operator $\mathscr{D}^{n}$ is defined by $\mathscr{D}^{0} f=f, \mathscr{D}^{n} f=f^{(n)}, f \in H(\mathbb{D})$.

The weighted differentiation composition operator, denoted by $\mathscr{D}_{\varphi, u}^{n}$, is defined as follows $[12,13]$ :

$$
\mathscr{D}_{\varphi, u}^{n} f(z)=u(z) f^{(n)}(\varphi(z)), \quad f \in H(\mathbb{D}),
$$

where $u \in H(\mathbb{D})$ and $\varphi$ is a nonconstant holomorphic selfmap of $\mathbb{D}$.

If $n=0$, then $\mathscr{D}_{\varphi, u}^{n}$ becomes the weighted composition operator $u C_{\varphi}$, defined by

$$
u C_{\varphi} f(z)=u(z) f(\varphi(z)), \quad z \in \mathbb{D},
$$

which, for $u(z) \equiv 1$, is reduced to the composition operator $C_{\varphi}$ for some recent articles on weighted composition operators on some $H^{\infty}$-type spaces, for example, [14-16] and references therein. If $n=1, u(z)=\varphi^{\prime}(z)$, then $\mathscr{D}_{\varphi, u}^{n}=$ $\mathscr{D} C_{\varphi}$, which was studied in [17-21]. When $n=1, u(z) \equiv$ 1 , then $\mathscr{D}_{\varphi, u}^{n}=C_{\varphi} \mathscr{D}$, which was studied in $[17,19]$. If $n=1, \varphi(z)=z$, then $\mathscr{D}_{\varphi, u}^{n}=M_{u} \mathscr{D}$, that is, the product of differentiation operator and multiplication operator $M_{u}$ defined by $M_{u} f=u f$. Zhu in [13] completely characterized the boundedness and compactness of linear operators which are obtained by taking products of differentiation, composition, and multiplication operators from Bergman type spaces to Bers spaces. Stević in [12] studied the boundedness and compactness of the weighted differentiation composition operator $\mathscr{D}_{\varphi, u}^{n}$ from mixed-norm spaces to weighted-type spaces or the little weighted-type space (see also [22-24]). Zhu in [25] studied the boundedness and compactness of the generalized weighted composition operator on weighted Bergman spaces. Yang in [21] studied the boundedness and compactness of the operator $C_{\varphi} \mathscr{D}$ and $\mathscr{D} C_{\varphi}$ from $Q_{K}(p, q)$ to $\mathscr{B}_{\mu}$ and $\mathscr{B}_{\mu, 0}$ spaces. Liu and Yu in [18] studied the boundedness and compactness of the operator $\mathscr{D} C_{\varphi}$ between $H^{\infty}$ and Zygmund spaces. Ye and Zhou in [26] studied the boundedness and compactness of the weighted composition operators from Hardy to Zygmund type spaces. Stević in [27] studied the boundedness and compactness of the generalized composition operator from mixed-norm space to the Blochtype space, the little Bloch-type space, the Zygmund space, and the little Zygmund space. For other recently introduced products of operators on spaces of holomorphic functions see $[13,16]$. Motivated by the results $[12,18,23,24,27]$, we consider the boundedness and compactness of the operators $\mathscr{D}_{\varphi, u}^{n}$ from the logarithmic Bloch spaces to the Zygmund-type spaces and the little Zygmund-type spaces. For the proof, we need different test functions and some complex calculations kills.

Throughout this paper, we will use the letter $C$ to denote a positive constant that can change its value at each occurrence.

\section{Auxiliary Results}

Here we prove and quote some auxiliary results which will be used in the proofs of the main results in this paper.

Lemma 1. Let $n$ be a positive integer. Suppose $f \in \mathscr{B}_{\log }$; there exists a constant $C$ such that

$$
\left|f^{(n)}(z)\right| \leq \frac{C\|f\|_{\mathscr{B}_{\log }}}{\left(1-|z|^{2}\right)^{n} \log (2 /(1-|z|))}, \quad z \in \mathbb{D},
$$

Proof. We use induction on $n$. Using the definition of the logarithmic Bloch spaces we have

$$
\left|f^{\prime}(z)\right| \leq \frac{C\|f\|_{\mathscr{B}_{\log }}}{\left(1-|z|^{2}\right) \log (2 /(1-|z|))}
$$

the case holds for $n=1$. Assume the case $n=k$ holds; since

$$
\frac{1}{2 \pi} \int_{0}^{2 \pi} \frac{1}{\left|e^{i \theta}-z\right|^{2}} d \theta=\frac{1}{1-|z|^{2}}, \quad z \in \mathbb{D}
$$

let $\rho=(1+|z|) / 2<1$; then we have $|z / \rho|=2|z| /(1+|z|)<1$, so

$$
\frac{1}{2 \pi} \int_{0}^{2 \pi} \frac{1}{\left|\rho e^{i \theta}-z\right|^{2}} d \theta=\frac{1}{\rho^{2}-|z|^{2}}, \quad z \in \mathbb{D} .
$$

By the Cauchy integral formula we obtain

$$
\left|f^{(k+1)}(z)\right|=\frac{1}{2 \pi}\left|\int_{0}^{2 \pi} \frac{f^{(k)}\left(\rho e^{i \theta}\right)}{\left(\rho e^{i \theta}-z\right)^{2}} \rho e^{i \theta} d \theta\right|
$$




$$
\begin{aligned}
& \leq \frac{1}{2 \pi} \int_{0}^{2 \pi}\left|\frac{f^{(k)}\left(\rho e^{i \theta}\right)}{\left(\rho e^{i \theta}-z\right)^{2}} \rho e^{i \theta}\right| d \theta \\
& \leq \frac{C\|f\|_{\mathscr{B}_{\log }}}{\left(1-\rho^{2}\right)^{k}(\log (2 /(1-\rho)))} \\
& \times \frac{1}{2 \pi} \int_{0}^{2 \pi} \frac{\rho}{\left|\rho e^{i \theta}-z\right|^{2}} d \theta \\
& =\frac{C\|f\|_{\mathscr{B}_{\log }}}{\left(1-\rho^{2}\right)^{k}(\log (2 /(1-\rho)))} \frac{\rho}{\rho^{2}-|z|^{2}} \\
& =\frac{C\|f\|_{\mathscr{B}_{\log }}}{\left(1-\rho^{2}\right)^{k}(\log (2 /(1-\rho)))} \frac{\rho}{(\rho+|z|)(\rho-|z|)} \\
& \leq \frac{C\|f\|_{\mathscr{B}_{\log }}}{\left(1-\rho^{2}\right)^{k}(\log (2 /(1-\rho)))} \frac{1}{\rho-|z|} \\
& =\frac{C\|f\|_{\mathscr{R}_{\log }}}{\left(1-\rho^{2}\right)^{k}(\log (2 /(1-\rho)))} \frac{1}{1-\rho} \\
& \leq \frac{C\|f\|_{\mathscr{B}_{\log }}}{\left(1-\rho^{2}\right)^{k}(\log (2 /(1-\rho)))} \frac{2}{(1-\rho)(1+\rho)} \\
& \leq \frac{2 C\|f\|_{\mathscr{B}_{\log }}}{\left(1-\rho^{2}\right)^{k+1}(\log (2 /(1-\rho)))} .
\end{aligned}
$$

Note that

$$
\begin{gathered}
\frac{1}{4}(1-|z|) \leq 1-\rho=\frac{1}{2}(1-|z|) \leq 1-|z|, \\
\frac{1}{2}(1-|z|) \leq 1-\rho^{2}=(1+\rho)(1-\rho) \leq 1-|z| ;
\end{gathered}
$$

we have

$$
\left|f^{(k+1)}(z)\right| \leq \frac{C\|f\|_{\mathscr{B}_{\log }}}{\left(1-|z|^{2}\right)^{k+1} \log (2 /(1-|z|))},
$$

for every $z \in \mathbb{D}$. Hence the case $n=k+1$ holds. The desired result follows. The proof of this lemma is complete.

Lemma 2 (see $[4,28])$. Let

$$
g_{t}(z)=\frac{(1-|z|) \log (2 /(1-|z|))}{(1-|t z|) \log (2 /(1-|t z|))}, \quad t \in[0,1], z \in \mathbb{D}
$$

then $\left|g_{t}(z)\right|<2$.

The following criterion for the compactness is a useful tool and it follows from standard arguments (e.g., [29, Proposition 3.11] or [30, Lemma 2.10]).
Lemma 3. Let $u \in H(\mathbb{D})$, and let $n$ be a nonnegative integer, $\varphi$ a holomorphic self-map of $\mathbb{D}$, and $\mu$ a weight. Then $\mathscr{D}_{\varphi, u}^{n}: \mathscr{B}_{\log }\left(\mathscr{B}_{\log , 0}\right) \rightarrow \mathscr{Z}_{\mu}$ is compact if and only if $\mathscr{D}_{\varphi, u}^{n}: \mathscr{B}_{\log }\left(\mathscr{B}_{\log , 0}\right) \rightarrow \mathscr{Z}_{\mu}$ is bounded and, for any bounded sequence $\left\{f_{k}\right\}$ in $\mathscr{B}_{\log }\left(\mathscr{B}_{\log , 0}\right)$ which converges to zero uniformly on compact subsets of $\mathbb{D}$ as $k \rightarrow \infty$, we have $\left\|\mathscr{D}_{\varphi, u}^{n} f_{k}\right\|_{\mathscr{E}_{\mu}} \rightarrow 0$ as $k \rightarrow \infty$.

Lemma 4. A closed set $K$ in $\mathscr{Z}_{\mu, 0}$ is compact if and only if $K$ is bounded and satisfies

$$
\lim _{|z| \rightarrow 1_{f \in K}} \sup _{f \in(z)} \mu f^{\prime \prime}(z) \mid=0
$$

The proof is similar to that of Lemma 1 in [31]; hence we omit it.

\section{Boundedness and Compactness of $\mathscr{D}_{\varphi, u}^{n}$ from $\mathscr{B}_{\log }\left(\mathscr{B}_{\log , 0}\right)$ to $\mathscr{Z}_{\mu}\left(\mathscr{Z}_{\mu, 0}\right)$ Spaces}

In this section, we study the boundedness and compactness of $\mathscr{D}_{\varphi, u}^{n}: \mathscr{B}_{\log }\left(\mathscr{B}_{\log , 0}\right) \rightarrow \mathscr{Z}_{\mu}\left(\mathscr{Z}_{\mu, 0}\right)$.

Theorem 5. Let $u \in H(\mathbb{D})$, and let $n$ be a nonnegative integer, $\varphi$ a holomorphic self-map of $\mathbb{D}$, and $\mu$ a weight. Then the following statements are equivalent:

(1) $\mathscr{D}_{\varphi, u}^{n}: \mathscr{B}_{\log } \rightarrow \mathscr{Z}_{\mu}$ is bounded;

(2) $\mathscr{D}_{\varphi, u}^{n}: \mathscr{B}_{\log , 0} \rightarrow \mathscr{Z}_{\mu}$ is bounded;

(3)

$$
\begin{gathered}
\sup _{z \in \mathbb{D}} \frac{\mu(z)\left|u^{\prime \prime}(z)\right|}{\left(1-|\varphi(z)|^{2}\right)^{n} \log (2 /(1-|\varphi(z)|))}<\infty, \\
\sup _{z \in \mathbb{D}} \frac{\mu(z)\left|2 u^{\prime}(z) \varphi^{\prime}(z)+u(z) \varphi^{\prime \prime}(z)\right|}{\left(1-|\varphi(z)|^{2}\right)^{n+1} \log (2 /(1-|\varphi(z)|))}<\infty, \\
\sup _{z \in \mathbb{D}} \frac{\mu(z)\left|u(z)\left(\varphi^{\prime}(z)\right)^{2}\right|}{\left(1-|\varphi(z)|^{2}\right)^{n+2} \log (2 /(1-|\varphi(z)|))}<\infty .
\end{gathered}
$$

Proof. (3) $\Rightarrow$ (1). Suppose that (17), (18), and (19) hold. Then, for every $z \in \mathbb{D}$ and $f \in \mathscr{B}_{\text {log }}$, by Lemma 1 , we have

$$
\begin{aligned}
& \mu(z)\left|\left(\mathscr{D}_{\varphi, u}^{n} f\right)^{\prime \prime}(z)\right| \\
& =\mu(z) \mid u^{\prime \prime}(z) f^{(n)}(\varphi(z)) \\
& +\left(2 u^{\prime}(z) \varphi^{\prime}(z)+u(z) \varphi^{\prime \prime}(z)\right) f^{(n+1)}(\varphi(z)) \\
& +u(z)\left(\varphi^{\prime}(z)\right)^{2} f^{(n+2)}(\varphi(z)) \mid
\end{aligned}
$$




$$
\begin{aligned}
& \leq \mu(z)\left|u^{\prime \prime}(z)\right|\left|f^{(n)}(\varphi(z))\right| \\
& \quad+\mu(z)\left|2 u^{\prime}(z) \varphi^{\prime}(z)+u(z) \varphi^{\prime \prime}(z)\right|\left|f^{(n+1)}(\varphi(z))\right| \\
& \quad+\mu(z)\left|u(z)\left(\varphi^{\prime}(z)\right)^{2}\right|\left|f^{(n+2)}(\varphi(z))\right| \\
& \leq C\|f\|_{\mathscr{B}_{\log }} \frac{\mu(z)\left|u^{\prime \prime}(z)\right|}{\left(1-|\varphi(z)|^{2}\right)^{n} \log (2 /(1-|\varphi(z)|))} \\
& \quad+C\|f\|_{\mathscr{B}_{\log }} \frac{\mu(z)\left|2 u^{\prime}(z) \varphi^{\prime}(z)+u(z) \varphi^{\prime \prime}(z)\right|}{\left(1-|\varphi(z)|^{2}\right)^{n+1} \log (2 /(1-|\varphi(z)|))} \\
& \quad+C\|f\|_{\mathscr{B}_{\log }} \frac{\mu(z)\left|u(z)\left(\varphi^{\prime}(z)\right)^{2}\right|}{\left(1-|\varphi(z)|^{2}\right)^{n+2} \log (2 /(1-|\varphi(z)|))} \\
& \quad+C\|f\|_{\mathscr{B}_{\log } \cdot}
\end{aligned}
$$

On the other hand, we have

$$
\begin{aligned}
& \left|\left(\mathscr{D}_{\varphi, u}^{n} f\right)(0)\right| \\
& \quad=\left|u(0) f^{(n)}(\varphi(0))\right| \\
& \leq C \frac{|u(0)|}{\left(1-|\varphi(0)|^{2}\right)^{n} \log (2 /(1-|\varphi(0)|))}\|f\|_{\mathscr{B}_{\log }} \\
& \left|\left(\mathscr{D}_{\varphi, u}^{n} f\right)^{\prime}(0)\right| \\
& =\left|u(0)^{\prime} f^{(n)}(\varphi(0))+u(0) f^{(n+1)}(\varphi(0)) \varphi^{\prime}(0)\right| \\
& \leq C \frac{\left|u^{\prime}(0)\right|}{\left(1-|\varphi(0)|^{2}\right)^{n} \log (2 /(1-|\varphi(0)|))}\|f\|_{\mathscr{B}_{\log }} \\
& \quad+C \frac{\left|u(0) \varphi^{\prime}(0)\right|}{\left(1-|\varphi(0)|^{2}\right)^{n+1} \log (2 /(1-|\varphi(0)|))}\|f\|_{\mathscr{B}_{\log }} .
\end{aligned}
$$

Applying conditions (20) and (21), we deduce that the operator $\mathscr{D}_{\varphi, u}^{n}: \mathscr{B}_{\log } \rightarrow \mathscr{Z}_{\mu}$ is bounded.

$(1) \Rightarrow(2)$. This implication is clear.

$(2) \Rightarrow(3)$. Assume that $\mathscr{D}_{\varphi, u}^{n}: \mathscr{B}_{\log , 0} \rightarrow \mathscr{Z}_{\mu}$ is bounded; that is, there exists a constant $C$, such that

$$
\left\|\mathscr{D}_{\varphi, u}^{n} f\right\|_{\mathscr{F}_{\mu}} \leq C\|f\|_{\mathscr{B}_{\log }},
$$

for all $f \in \mathscr{B}_{\log , 0}$. For $f(z)=z^{n} / n ! \in \mathscr{B}_{\log , 0}$, we have that

$$
K_{1}:=\sup _{z \in \mathbb{D}} \mu(z)\left|u^{\prime \prime}(z)\right|<\infty .
$$

Taking $f(z)=z^{n+1} /(n+1) ! \in \mathscr{B}_{\log , 0}$; we have that

$$
\sup _{z \in \mathbb{D}} \mu(z)\left|u^{\prime \prime}(z) \varphi(z)+2 u^{\prime}(z) \varphi^{\prime}(z)+u(z) \varphi^{\prime \prime}(z)\right|<\infty
$$

By (23), (24), and the boundedness of the function $\varphi(z)$, we get

$$
K_{2}:=\sup _{z \in \mathbb{D}} \mu(z)\left|2 u^{\prime}(z) \varphi^{\prime}(z)+u(z) \varphi^{\prime \prime}(z)\right|<\infty .
$$

In the same way, taking $f(z)=z^{n+2} /(n+2) ! \in \mathscr{B}_{\text {log, } 0}$, we have that

$$
\begin{aligned}
\sup _{z \in \mathbb{D}} \mu(z) \mid u^{\prime \prime}(z)(\varphi(z))^{2} & \\
& +2\left(2 u^{\prime}(z) \varphi^{\prime}(z)+u(z) \varphi^{\prime \prime}(z)\right) \varphi(z) \\
& +2 u(z)\left(\varphi^{\prime}(z)\right)^{2} \mid<\infty .
\end{aligned}
$$

By (23), (25), (26), and the boundedness of the function $\varphi(z)$, we have that

$$
K_{3}:=\sup _{z \in \mathbb{D}} \mu(z)\left|u(z)\left(\varphi^{\prime}(z)\right)^{2}\right|<\infty .
$$

For a fixed $\omega \in \mathbb{D}$, set

$$
\begin{aligned}
f_{\omega}(z)= & (n+2)(n+3) \frac{1-|\varphi(\omega)|^{2}}{(1-z \overline{\varphi(\omega)}) \log (2 /(1-|\varphi(\omega)|))} \\
& -2(n+3) \frac{\left(1-|\varphi(\omega)|^{2}\right)^{2}}{(1-z \overline{\varphi(\omega)})^{2} \log (2 /(1-|\varphi(\omega)|))} \\
& +2 \frac{\left(1-|\varphi(\omega)|^{2}\right)^{3}}{(1-z \overline{\varphi(\omega)})^{3} \log (2 /(1-|\varphi(\omega)|))}
\end{aligned}
$$

We get that

$$
\begin{aligned}
& f_{\omega}^{(n)}(z) \\
& =\frac{(n+3) !}{n+1} \frac{\left(1-|\varphi(\omega)|^{2}\right)(\overline{\varphi(\omega)})^{n}}{(1-z \overline{\varphi(\omega)})^{n+1} \log (2 /(1-|\varphi(\omega)|))} \\
& \quad-2(n+3) \cdot(n+1) ! \frac{\left(1-|\varphi(\omega)|^{2}\right)^{2}(\overline{\varphi(\omega)})^{n}}{(1-z \overline{\varphi(\omega)})^{n+2} \log (2 /(1-|\varphi(\omega)|))}
\end{aligned}
$$$$
+(n+2) ! \frac{\left(1-|\varphi(\omega)|^{2}\right)^{3}(\overline{\varphi(\omega)})^{n}}{(1-z \overline{\varphi(\omega)})^{n+3} \log (2 /(1-|\varphi(\omega)|))} ;
$$

$f_{\omega}^{(n+1)}(z)$

$=(n+3) ! \frac{\left(1-|\varphi(\omega)|^{2}\right)(\overline{\varphi(\omega)})^{n+1}}{(1-z \overline{\varphi(\omega)})^{n+2} \log (2 /(1-|\varphi(\omega)|))}$ 


$$
\begin{aligned}
& -2 \cdot(n+3) ! \frac{\left(1-|\varphi(\omega)|^{2}\right)^{2}(\overline{\varphi(\omega)})^{n+1}}{(1-z \overline{\varphi(\omega)})^{n+3} \log (2 /(1-|\varphi(\omega)|))} \\
& +(n+3) ! \frac{\left(1-|\varphi(\omega)|^{2}\right)^{3}(\overline{\varphi(\omega)})^{n+1}}{(1-z \overline{\varphi(\omega)})^{n+4} \log (2 /(1-|\varphi(\omega)|))} ; \\
& f_{\omega}^{(n+2)}(z) \\
& =(n+2) \cdot(n+3) ! \frac{\left(1-|\varphi(\omega)|^{2}\right)(\overline{\varphi(\omega)})^{n+2}}{(1-z \overline{\varphi(\omega)})^{n+3} \log (2 /(1-|\varphi(\omega)|))} \\
& -2(n+3) \cdot(n+3) ! \frac{\left(1-|\varphi(\omega)|^{2}\right)^{2}(\overline{\varphi(\omega)})^{n+2}}{(1-z \overline{\varphi(\omega)})^{n+4} \log (2 /(1-|\varphi(\omega)|))} \\
& +(n+4) ! \frac{\left(1-|\varphi(\omega)|^{2}\right)^{3}(\overline{\varphi(\omega)})^{n+2}}{(1-z \overline{\varphi(\omega)})^{n+5} \log (2 /(1-|\varphi(\omega)|))} .
\end{aligned}
$$

By Lemma 2 we have

$$
\begin{aligned}
& \sup _{z \in \mathbb{D}}\left(1-|z|^{2}\right)\left(\log \frac{2}{1-|z|}\right)\left|f_{\omega}^{\prime}(z)\right| \\
& \leq \sup _{z \in \mathbb{D}}\left(1-|z|^{2}\right)\left(\log \frac{2}{1-|z|}\right) \\
& \times\left|\frac{(n+2)(n+3)\left(1-|\varphi(\omega)|^{2}\right) \overline{\varphi(\omega)}}{(1-z \overline{\varphi(\omega)})^{2} \log (2 /(1-|\varphi(\omega)|))}\right| \\
& +\sup _{z \in \mathbb{D}}\left(1-|z|^{2}\right)\left(\log \frac{2}{1-|z|}\right) \\
& \times\left|\frac{4(n+3)\left(1-|\varphi(\omega)|^{2}\right)^{2} \overline{\varphi(\omega)}}{(1-z \overline{\varphi(\omega)})^{3} \log (2 /(1-|\varphi(\omega)|))}\right| \\
& +\sup _{z \in \mathbb{D}}\left(1-|z|^{2}\right)\left(\log \frac{2}{1-|z|}\right) \\
& \times\left|\frac{6\left(1-|\varphi(\omega)|^{2}\right)^{3} \overline{\varphi(\omega)}}{(1-z \overline{\varphi(\omega)})^{4} \log (2 /(1-|\varphi(\omega)|))}\right| \\
& \leq 4(n+2)(n+3) \sup _{z \in \mathbb{D}}(1-|z|)\left(\log \frac{2}{1-|z|}\right) \\
& \times \frac{(1-|\varphi(\omega)|)}{(1-|\overline{\varphi(\omega)}|)(1-|z \overline{\varphi(\omega)}|) \log (2 /(1-|\varphi(\omega)|))} \\
& +32(n+3) \sup _{z \in \mathbb{D}}(1-|z|)\left(\log \frac{2}{1-|z|}\right)
\end{aligned}
$$

$$
\begin{aligned}
& \times \frac{(1-|\varphi(\omega)|)^{2}}{(1-|\overline{\varphi(\omega)}|)^{2}(1-|z \overline{\varphi(\omega)}|) \log (2 /(1-|\varphi(\omega)|))} \\
+ & 96 \sup _{z \in \mathbb{D}}(1-|z|)\left(\log \frac{2}{1-|z|}\right) \\
& \times \frac{(1-|\varphi(\omega)|)^{3}}{(1-|\overline{\varphi(\omega)}|)^{3}(1-|z \overline{\varphi(\omega)}|) \log (2 /(1-|\varphi(\omega)|))} \\
= & (n+2)(n+3) \sup _{z \in \mathbb{D}} \overline{(1-|z \overline{\varphi(\omega)}|)(\log (2 /(1-|z \overline{\varphi(\omega)}|)))} \\
\times & \frac{\log (2 /(1-|z \overline{\varphi(\omega)}|))}{\log (2 /(1-|\varphi(\omega)|))} \\
+ & 32(n+3) \sup _{z \in \mathbb{D}} \frac{(1-|z|)(\log (2 /(1-|z|)))}{(1-|\overline{\varphi(\omega)}|)(\log (2 /(1-|z \overline{\varphi(\omega)}|)))} \\
& \times \frac{\log (2 /(1-|z \overline{\varphi(\omega)}|))}{\log (2 /(1-|\varphi(\omega)|))}
\end{aligned}
$$$$
+96 \sup _{z \in \mathbb{D}} \frac{(1-|z|)(\log (2 /(1-|z|)))}{(1-|z \overline{\varphi(\omega)}|)(\log (2 /(1-|z \overline{\varphi(\omega)}|)))}
$$$$
\times \frac{\log (2 /(1-|z \overline{\varphi(\omega)}|))}{\log (2 /(1-|\varphi(\omega)|))}
$$$$
\leq 4(n+2)(n+3)
$$$$
\times \sup _{z \in \mathbb{D}} \frac{(1-|z|)(\log (2 /(1-|z|)))}{(1-|z \overline{\varphi(\omega)}|)(\log (2 /(1-|z \overline{\varphi(\omega)}|)))}
$$$$
\times \frac{\log (2 /(1-|\overline{\varphi(\omega)}|))}{\log (2 /(1-|\varphi(\omega)|))}
$$$$
+32(n+3) \sup _{z \in \mathbb{D}} \frac{(1-|z|)(\log (2 /(1-|z|)))}{(1-|z \overline{\varphi(\omega)}|)(\log (2 /(1-|z \overline{\varphi(\omega)}|)))}
$$$$
\times \frac{\log (2 /(1-|\overline{\varphi(\omega)}|))}{\log (2 /(1-|\varphi(\omega)|))}
$$$$
+96 \sup _{z \in \mathbb{D}} \frac{(1-|z|)(\log (2 /(1-|z|)))}{(1-|z \overline{\varphi(\omega)}|)(\log (2 /(1-|z \overline{\varphi(\omega)}|)))}
$$$$
\times \frac{\log (2 /(1-|\overline{\varphi(\omega)}|))}{\log (2 /(1-|\varphi(\omega)|))}
$$$$
\leq 8(n+2)(n+3)+64(n+3)+192
$$$$
=84 n^{2}+104 n+432 \text {. }
$$

Hence, $f_{\omega} \in \mathscr{B}_{\log }$ and $\sup _{\omega \in \mathbb{D}}\left\|f_{\omega}\right\|_{\mathscr{B}_{\log }} \leq C$. 
On the other hand for each fix $\omega \in \mathbb{D}$, by (30), we obtain that

$$
\left(1-|z|^{2}\right)\left(\log \frac{2}{1-|z|}\right)\left|f_{\omega}^{\prime}(z)\right| \longrightarrow 0, \quad(\text { as }|z| \longrightarrow 1)
$$

it follows that $f_{\omega} \in \mathscr{B}_{\log , 0}$ for each fix $\omega \in \mathbb{D}$. From (29), we have $f_{\omega}^{(n+1)}(\varphi(\omega))=f_{\omega}^{(n+2)}(\varphi(\omega))=0$ and

$$
f_{\omega}^{(n)}(\varphi(\omega))=2 \cdot n ! \frac{(\overline{\varphi(\omega)})^{n}}{\left(1-|\varphi(\omega)|^{2}\right)^{n} \log (2 /(1-|\varphi(\omega)|))}
$$

Hence

$$
\begin{aligned}
& C \geq\left\|\mathscr{D}_{\varphi, u}^{n} f_{\omega}\right\|_{\mathscr{Z}_{\mu}} \\
& \geq \sup _{z \in \mathbb{D}} \mu(z)\left|\left(\mathscr{D}_{\varphi, u}^{n} f_{\omega}\right)^{\prime \prime}(z)\right| \\
& =\sup _{z \in \mathbb{D}} \mu(z) \mid u^{\prime \prime}(z) f_{\omega}^{(n)}(\varphi(z)) \\
& +\left(2 u^{\prime}(z) \varphi^{\prime}(z)+u(z) \varphi^{\prime \prime}(z)\right) f_{\omega}^{(n+1)}(\varphi(z)) \\
& +u(z)\left(\varphi^{\prime}(z)\right)^{2} f_{\omega}^{(n+2)}(\varphi(z)) \\
& \geq \mu(\omega) \mid u^{\prime \prime}(\omega) f_{\omega}^{(n)}(\varphi(\omega)) \\
& +\left(2 u^{\prime}(\omega) \varphi^{\prime}(\omega)+u(\omega) \varphi^{\prime \prime}(\omega)\right) f_{\omega}^{(n+1)}(\varphi(\omega)) \\
& +u(\omega)\left(\varphi^{\prime}(\omega)\right)^{2} f_{\omega}^{(n+2)}(\varphi(\omega)) \\
& =2 \cdot n ! \frac{\mu(\omega)\left|u^{\prime \prime}(\omega)\right||\overline{\varphi(\omega)}|^{n}}{\left(1-|\varphi(\omega)|^{2}\right)^{n} \log (2 /(1-|\varphi(\omega)|))} .
\end{aligned}
$$

By (33), we obtain that

$$
\begin{aligned}
& \sup _{1 / 2<|\varphi(\omega)|<1} \frac{\mu(\omega)\left|u^{\prime \prime}(\omega)\right|}{\left(1-|\varphi(\omega)|^{2}\right)^{n} \log (2 /(1-|\varphi(\omega)|))} \\
& \leq 2^{n+1} \cdot n ! \sup _{1 / 2<|\varphi(\omega)|<1} \frac{\mu(\omega)\left|u^{\prime \prime}(\omega)\right||\overline{\varphi(\omega)}|^{n}}{\left(1-|\varphi(\omega)|^{2}\right)^{n} \log (2 /(1-|\varphi(\omega)|))} \\
& \leq 2^{n+1} \cdot n ! \sup _{\omega \in \mathbb{D}} \frac{\mu(\omega)\left|u^{\prime \prime}(\omega)\right||\overline{\varphi(\omega)}|^{n}}{\left(1-|\varphi(\omega)|^{2}\right)^{n} \log (2 /(1-|\varphi(\omega)|))} \\
& \leq C 2^{n}<\infty .
\end{aligned}
$$

And from (23), we have

$$
\begin{aligned}
& \sup _{|\varphi(\omega)| \leq 1 / 2} \frac{\mu(\omega)\left|u^{\prime \prime}(\omega)\right|}{\left(1-|\varphi(\omega)|^{2}\right)^{n} \log (2 /(1-|\varphi(\omega)|))} \\
& \quad \leq \sup _{|\varphi(\omega)| \leq 1 / 2} \frac{\mu(\omega)\left|u^{\prime \prime}(\omega)\right|}{\left(1-|\varphi(\omega)|^{2}\right)^{n} \log 2} \\
& \quad \leq\left(\frac{4}{3}\right)^{n} \frac{1}{\log 2} \sup _{|\varphi(\omega)| \leq 1 / 2} \mu(\omega)\left|u^{\prime \prime}(\omega)\right| \\
& \quad \leq\left(\frac{4}{3}\right)^{n} \frac{K_{1}}{\log 2}<\infty .
\end{aligned}
$$

Thus combining (35) with (34) we get the condition (17). For a fixed $\omega \in \mathbb{D}$, set

$$
\begin{aligned}
g_{\omega}(z)= & (n+1)(n+3) \frac{1-|\varphi(\omega)|^{2}}{(1-z \overline{\varphi(\omega)}) \log (2 /(1-|\varphi(\omega)|))} \\
& -(2 n+5) \frac{\left(1-|\varphi(\omega)|^{2}\right)^{2}}{(1-z \overline{\varphi(\omega)})^{2} \log (2 /(1-|\varphi(\omega)|))} \\
& +2 \frac{\left(1-|\varphi(\omega)|^{2}\right)^{3}}{(1-z \overline{\varphi(\omega)})^{3} \log (2 /(1-|\varphi(\omega)|))}
\end{aligned}
$$

It is easy to see that

$$
\begin{aligned}
& g_{\omega}^{(n)}(z) \\
& =(n+3) \cdot(n+1) ! \frac{\left(1-|\varphi(\omega)|^{2}\right)(\overline{\varphi(\omega)})^{n}}{(1-z \overline{\varphi(\omega)})^{n+1} \log (2 /(1-|\varphi(\omega)|))} \\
& \quad-(2 n+5) \cdot(n+1) ! \frac{\left(1-|\varphi(\omega)|^{2}\right)^{2}(\overline{\varphi(\omega)})^{n}}{(1-z \overline{\varphi(\omega)})^{n+2} \log (2 /(1-|\varphi(\omega)|))} \\
& \quad+(n+2) ! \frac{(1-z \overline{\varphi(\omega)})^{n+3} \log (2 /(1-|\varphi(\omega)|))}{(n+1)}(z) \\
& \left.\left.g_{\omega}^{2}\right|^{3(\omega)}\right)^{n} \\
& =\frac{(n+1) \cdot(n+3) !}{(n+2)} \frac{\left(1-|\varphi(\omega)|^{2}\right)(\overline{\varphi(\omega)})^{n+1}}{\left(1-\overline{\left.\varphi_{(\omega)}\right)^{n+2}} \log (2 /(1-|\varphi(\omega)|))\right.} \\
& \quad-(2 n+5) \cdot(n+2) ! \frac{\left(1-|\varphi(\omega)|^{2}\right)^{2}(\overline{\varphi(\omega)})^{n+1}}{(1-z \overline{\varphi(\omega)})^{n+3} \log (2 /(1-|\varphi(\omega)|))}
\end{aligned}
$$




$$
\begin{aligned}
& +(n+3) ! \frac{\left(1-|\varphi(\omega)|^{2}\right)^{3}(\overline{\varphi(\omega)})^{n+1}}{(1-z \overline{\varphi(\omega)})^{n+4} \log (2 /(1-|\varphi(\omega)|))} ; \\
& g_{\omega}^{(n+2)}(z) \\
& =(n+1) \cdot(n+3) ! \frac{\left(1-|\varphi(\omega)|^{2}\right)(\overline{\varphi(\omega)})^{n+2}}{(1-z \overline{\varphi(\omega)})^{n+3} \log (2 /(1-|\varphi(\omega)|))} \\
& -(2 n+5) \cdot(n+3) ! \frac{\left(1-|\varphi(\omega)|^{2}\right)^{2}(\overline{\varphi(\omega)})}{(1-z \overline{\varphi(\omega)})^{n+4} \log (2 /(1-|\varphi(\omega)|))} \\
& +(n+4) ! \frac{\left(1-|\varphi(\omega)|^{2}\right)^{3}(\overline{\varphi(\omega)})^{n+2}}{(1-z \overline{\varphi(\omega)})^{n+5} \log (2 /(1-|\varphi(\omega)|))} .
\end{aligned}
$$

Using Lemma 2, we easily get that $g_{\omega} \in \mathscr{B}_{\log , 0}$ and $\sup _{\omega \in \mathbb{D}}\left\|g_{\omega}\right\|_{\mathscr{R}_{\log }} \leq C$ with a direct calculation. From (37), we have $g_{\omega}^{(n)}(\varphi(\omega))=g_{\omega}^{(n+2)}(\varphi(\omega))=0$,

$$
\begin{aligned}
& g_{\omega}^{(n+1)}(\varphi(\omega)) \\
& \quad=-(n+1) ! \frac{(\overline{\varphi(\omega)})^{n+1}}{\left(1-|\varphi(\omega)|^{2}\right)^{n+1} \log (2 /(1-|\varphi(\omega)|))} .
\end{aligned}
$$

Hence

$$
\begin{aligned}
& C \geq\left\|\mathscr{D}_{\varphi, u}^{n} g_{\omega}\right\|_{\mathscr{X}_{\mu}} \\
& \geq \sup _{z \in \mathbb{D}} \mu(z)\left|\left(\mathscr{D}_{\varphi, u}^{n} g_{\omega}\right)^{\prime \prime}(z)\right| \\
& =\sup _{z \in \mathbb{D}} \mu(z) \mid u^{\prime \prime}(z) g_{\omega}^{(n)}(\varphi(z)) \\
& +\left(2 u^{\prime}(z) \varphi^{\prime}(z)+u(z) \varphi^{\prime \prime}(z)\right) g_{\omega}^{(n+1)}(\varphi(z)) \\
& \quad+u(z)\left(\varphi^{\prime}(z)\right)^{2} g_{\omega}^{(n+2)}(\varphi(z)) \mid \\
& \geq \mu(\omega) \mid u^{\prime \prime}(\omega) g_{\omega}^{(n)}(\varphi(\omega)) \\
& +\left(2 u^{\prime}(\omega) \varphi^{\prime}(\omega)+u(\omega) \varphi^{\prime \prime}(\omega)\right) g_{\omega}^{(n+1)}(\varphi(\omega)) \\
& +u(\omega)\left(\varphi^{\prime}(\omega)\right)^{2} g_{\omega}^{(n+2)}(\varphi(\omega)) \mid \\
& =(n+1) ! \\
& \quad \frac{\mu(\omega)\left|2 u^{\prime}(\omega) \varphi^{\prime}(\omega)+u(\omega) \varphi^{\prime \prime}(\omega)\right||\overline{\varphi(\omega)}|^{n+1}}{\left(1-|\varphi(\omega)|^{2}\right)^{n+1} \log (2 /(1-|\varphi(\omega)|))} .
\end{aligned}
$$

From (39), we obtain that

$$
\begin{aligned}
& \sup _{1 / 2<|\varphi(\omega)|<1} \frac{\mu(\omega)\left|2 u^{\prime}(\omega) \varphi^{\prime}(\omega)+u(\omega) \varphi^{\prime \prime}(\omega)\right|}{\left(1-|\varphi(\omega)|^{2}\right)^{n+1} \log (2 /(1-|\varphi(\omega)|))} \\
& \leq 2^{n+1} \sup _{1 / 2<|\varphi(\omega)|<1}(n+1) ! \\
& \quad \times \frac{\mu(\omega)\left|2 u^{\prime}(\omega) \varphi^{\prime}(\omega)+u(\omega) \varphi^{\prime \prime}(\omega)\right||\overline{\varphi(\omega)}|^{n+1}}{\left(1-|\varphi(\omega)|^{2}\right)^{n+1} \log (2 /(1-|\varphi(\omega)|))} \\
& \leq 2^{n+1}(n+1) ! \\
& \quad \times \sup _{\omega \in \mathbb{D}} \frac{\mu(\omega)\left|2 u^{\prime}(\omega) \varphi^{\prime}(\omega)+u(\omega) \varphi^{\prime \prime}(\omega)\right||\overline{\varphi(\omega)}|^{n+1}}{\left(1-|\varphi(\omega)|^{2}\right)^{n+1} \log (2 /(1-|\varphi(\omega)|))} \\
& \leq C 2^{n+1}<\infty .
\end{aligned}
$$

By (25), we have

$$
\begin{aligned}
& \sup _{|\varphi(\omega)| \leq 1 / 2} \frac{\mu(\omega)\left|2 u^{\prime}(\omega) \varphi^{\prime}(\omega)+u(\omega) \varphi^{\prime \prime}(\omega)\right|}{\left(1-|\varphi(\omega)|^{2}\right)^{n+1} \log (2 /(1-|\varphi(\omega)|))} \\
& \leq \sup _{|\varphi(\omega)| \leq 1 / 2} \frac{\mu(\omega)\left|2 u^{\prime}(\omega) \varphi^{\prime}(\omega)+u(\omega) \varphi^{\prime \prime}(\omega)\right|}{\left(1-|\varphi(\omega)|^{2}\right)^{n+1} \log 2} \\
& \leq\left(\frac{4}{3}\right)^{n+1} \frac{1}{\log 2} \sup _{|\varphi(\omega)| \leq 1 / 2} \mu(\omega) \mid 2 u^{\prime}(\omega) \varphi^{\prime}(\omega) \\
& \quad+u(\omega) \varphi^{\prime \prime}(\omega) \mid \\
& \leq\left(\frac{4}{3}\right)^{n+1} \frac{K_{2}}{\log 2}<\infty .
\end{aligned}
$$

Thus combining (40) with (41) we get the condition (18).

Next, we prove (19). To see this, for a fixed $\omega \in \mathbb{D}$, put

$$
\begin{aligned}
h_{\omega}(z)= & (n+1)(n+2) \frac{1-|\varphi(\omega)|^{2}}{(1-z \overline{\varphi(\omega)}) \log (2 /(1-|\varphi(\omega)|))} \\
& -2(n+2) \frac{\left(1-|\varphi(\omega)|^{2}\right)^{2}}{(1-z \overline{\varphi(\omega)})^{2} \log (2 /(1-|\varphi(\omega)|))} \\
& +2 \frac{\left(1-|\varphi(\omega)|^{2}\right)^{3}}{(1-z \overline{\varphi(\omega)})^{3} \log (2 /(1-|\varphi(\omega)|))} .
\end{aligned}
$$


It is easy to see that

$$
\begin{aligned}
& h_{\omega}^{(n)}(z)=(n+2) ! \frac{\left(1-|\varphi(\omega)|^{2}\right)(\overline{\varphi(\omega)})^{n}}{(1-z \overline{\varphi(\omega)})^{n+1} \log (2 /(1-|\varphi(\omega)|))} \\
& -2 \cdot(n+2) ! \frac{\left(1-|\varphi(\omega)|^{2}\right)^{2}(\overline{\varphi(\omega)})^{n}}{(1-z \overline{\varphi(\omega)})^{n+2} \log (2 /(1-|\varphi(\omega)|))} \\
& +(n+2) ! \frac{\left(1-|\varphi(\omega)|^{2}\right)^{3}(\overline{\varphi(\omega)})^{n}}{(1-z \overline{\varphi(\omega)})^{n+3} \log (2 /(1-|\varphi(\omega)|))} ; \\
& h_{\omega}^{(n+1)}(z)=(n+1) \\
& \cdot(n+2) ! \frac{\left(1-|\varphi(\omega)|^{2}\right)(\overline{\varphi(\omega)})^{n+1}}{(1-z \overline{\varphi(\omega)})^{n+2} \log (2 /(1-|\varphi(\omega)|))} \\
& -2(n+2) \\
& \cdot(n+2) ! \frac{\left(1-|\varphi(\omega)|^{2}\right)^{2}(\overline{\varphi(\omega)})^{n+1}}{(1-z \overline{\varphi(\omega)})^{n+3} \log (2 /(1-|\varphi(\omega)|))} \\
& +(n+3) ! \frac{\left(1-|\varphi(\omega)|^{2}\right)^{3}(\overline{\varphi(\omega)})^{n+1}}{(1-z \overline{\varphi(\omega)})^{n+4} \log (2 /(1-|\varphi(\omega)|))} ; \\
& h_{\omega}^{(n+2)}(z)=(n+1)(n+2) \\
& \cdot(n+2) ! \frac{\left(1-|\varphi(\omega)|^{2}\right)(\overline{\varphi(\omega)})^{n+2}}{(1-z \overline{\varphi(\omega)})^{n+3} \log (2 /(1-|\varphi(\omega)|))} \\
& -2(n+2) \\
& \cdot(n+3) ! \frac{\left(1-|\varphi(\omega)|^{2}\right)^{2}(\overline{\varphi(\omega)})^{n+2}}{(1-z \overline{\varphi(\omega)})^{n+4} \log (2 /(1-|\varphi(\omega)|))} \\
& +(n+4) ! \frac{\left(1-|\varphi(\omega)|^{2}\right)^{3}(\overline{\varphi(\omega)})^{n+2}}{(1-z \overline{\varphi(\omega)})^{n+5} \log (2 /(1-|\varphi(\omega)|))}
\end{aligned}
$$

From Lemma 2 we obtain that $h_{\omega} \in \mathscr{B}_{\log , 0}$ and $\sup _{\omega \in \mathbb{D}}\left\|h_{\omega}\right\|_{\mathscr{B}_{\log }} \leq C$ with a direct calculation. From (43), we have $h_{\omega}^{(n)}(\varphi(\omega))=h_{\omega}^{(n+1)}(\varphi(\omega))=0$,

$$
\begin{aligned}
& h_{\omega}^{(n+2)}(\varphi(\omega)) \\
& \quad=2 \cdot(n+2) ! \frac{(\overline{\varphi(\omega)})^{n+2}}{\left(1-|\varphi(\omega)|^{2}\right)^{n+2} \log (2 /(1-|\varphi(\omega)|))} .
\end{aligned}
$$

Hence

$$
\begin{aligned}
& C \geq\left\|\mathscr{D}_{\varphi, u}^{n} h_{\omega}\right\|_{\mathscr{X}_{\mu}} \\
& \geq \sup _{z \in \mathbb{D}} \mu(z)\left|\left(\mathscr{D}_{\varphi, u}^{n} h_{\omega}\right)^{\prime \prime}(z)\right| \\
& =\sup _{z \in \mathbb{D}} \mu(z) \mid u^{\prime \prime}(z) h_{\omega}^{(n)}(\varphi(z)) \\
& +\left(2 u^{\prime}(z) \varphi^{\prime}(z)+u(z) \varphi^{\prime \prime}(z)\right) h_{\omega}^{(n+1)}(\varphi(z)) \\
& +u(z)\left(\varphi^{\prime}(z)\right)^{2} h_{\omega}^{(n+2)}(\varphi(z)) \\
& \geq \mu(\omega) \mid u^{\prime \prime}(\omega) h_{\omega}^{(n)}(\varphi(\omega)) \\
& +\left(2 u^{\prime}(\omega) \varphi^{\prime}(\omega)+u(\omega) \varphi^{\prime \prime}(\omega)\right) h_{\omega}^{(n+1)}(\varphi(\omega)) \\
& +u(\omega)\left(\varphi^{\prime}(\omega)\right)^{2} h_{\omega}^{(n+2)}(\varphi(\omega)) \\
& =2 \cdot(n+2) ! \frac{\mu(\omega)\left|u(\omega)\left(\varphi^{\prime}(\omega)\right)^{2}\right||\overline{\varphi(\omega)}|^{n+2}}{\left(1-|\varphi(\omega)|^{2}\right)^{n+2} \log (2 /(1-|\varphi(\omega)|))} .
\end{aligned}
$$

By (45), we obtain that

$$
\begin{aligned}
& \sup _{1 / 2<|\varphi(\omega)|<1} \frac{\mu(\omega)\left|u(\omega)\left(\varphi^{\prime}(\omega)\right)^{2}\right|}{\left(1-|\varphi(\omega)|^{2}\right)^{n+2} \log (2 /(1-|\varphi(\omega)|))} \\
& \leq 2^{n+2} \sup _{1 / 2<|\varphi(\omega)|<1} 2 \\
& \cdot(n+2) ! \frac{\mu(\omega)\left|u(\omega)\left(\varphi^{\prime}(\omega)\right)^{2}\right||\overline{\varphi(\omega)}|^{n+2}}{\left(1-|\varphi(\omega)|^{2}\right)^{n+2} \log (2 /(1-|\varphi(\omega)|))} \\
& \leq 2^{n+2} \sup _{\omega \in \mathbb{D}} \cdot(n+2) ! \frac{\mu(\omega)\left|u(\omega)\left(\varphi^{\prime}(\omega)\right)^{2}\right||\overline{\varphi(\omega)}|^{n+2}}{\left(1-|\varphi(\omega)|^{2}\right)^{n+2} \log (2 /(1-|\varphi(\omega)|))} \\
& \leq C 2^{n+2}<\infty .
\end{aligned}
$$

By (27), we have

$$
\begin{aligned}
& \sup _{|\varphi(\omega)| \leq 1 / 2} \frac{\mu(\omega)\left|u(\omega)\left(\varphi^{\prime}(\omega)\right)^{2}\right|}{\left(1-|\varphi(\omega)|^{2}\right)^{n+2} \log (2 /(1-|\varphi(\omega)|))} \\
& \quad \leq \sup _{|\varphi(\omega)| \leq 1 / 2} \frac{\mu(\omega)\left|u(\omega)\left(\varphi^{\prime}(\omega)\right)^{2}\right|}{\left(1-|\varphi(\omega)|^{2}\right)^{n+2} \log 2} \\
& \leq\left(\frac{4}{3}\right)^{n+2} \frac{1}{\log 2} \sup _{|\varphi(\omega)| \leq 1 / 2} \mu(\omega)\left|u(\omega)\left(\varphi^{\prime}(\omega)\right)^{2}\right| \\
& \leq\left(\frac{4}{3}\right)^{n+2} \frac{K_{3}}{\log 2}<\infty .
\end{aligned}
$$


Thus combining (47) with (46) we get the condition (19), finishing the proof of the theorem.

Theorem 6. Let $u \in H(\mathbb{D})$, and let $n$ be a nonnegative integer, $\varphi$ a holomorphic self-map of $\mathbb{D}$, and $\mu$ a weight. Then the following statements are equivalent:

(1) $\mathscr{D}_{\varphi, u}^{n}: \mathscr{B}_{\log } \rightarrow \mathscr{Z}_{\mu}$ is compact;

(2) $\mathscr{D}_{\varphi, u}^{n}: \mathscr{B}_{\log , 0} \rightarrow \mathscr{Z}_{\mu}$ is compact;

(3) $\mathscr{D}_{\varphi, u}^{n}: \mathscr{B}_{\log } \rightarrow \mathscr{Z}_{\mu}$ is bounded and

$$
\begin{gathered}
\lim _{|\varphi(z)| \rightarrow 1} \frac{\mu(z)\left|u^{\prime \prime}(z)\right|}{\left(1-|\varphi(z)|^{2}\right)^{n} \log (2 /(1-|\varphi(z)|))}=0, \\
\lim _{|\varphi(z)| \rightarrow 1} \frac{\mu(z)\left|2 u^{\prime}(z) \varphi^{\prime}(z)+u(z) \varphi^{\prime \prime}(z)\right|}{\left(1-|\varphi(z)|^{2}\right)^{n+1} \log (2 /(1-|\varphi(z)|))}=0, \\
\lim _{|\varphi(z)| \rightarrow 1} \frac{\mu(z)\left|u(z)\left(\varphi^{\prime}(z)\right)^{2}\right|}{\left(1-|\varphi(z)|^{2}\right)^{n+2} \log (2 /(1-|\varphi(z)|))}=0 .
\end{gathered}
$$

Proof. (3) $\Rightarrow$ (1). Assume that $\mathscr{D}_{\varphi, u}^{n}: \mathscr{B}_{\log } \rightarrow \mathscr{Z}_{\mu}$ is bounded and that conditions (48), (49), and (50) hold. For any bounded sequence $\left\{f_{k}\right\}$ in $\mathscr{B}_{\log }$ which converges to zero uniformly on compact subsets of $\mathbb{D}$. To establish the assertion, it suffices, in view of Lemma 3, to show that

$$
\left\|\mathscr{D}_{\varphi, u}^{n} f_{k}\right\|_{\mathscr{Z}_{\mu}} \longrightarrow 0, \quad \text { as } k \longrightarrow \infty \text {. }
$$

We assume that $\left\|f_{k}\right\|_{\mathscr{B}_{\log }} \leq 1$. From (48), (49), and (50) we have that, for any $\varepsilon>0$, there exists $\rho \in(0,1)$; when $\rho<$ $|\varphi(z)|<1$, we have

$$
\begin{gathered}
\frac{\mu(z)\left|u^{\prime \prime}(z)\right|}{\left(1-|\varphi(z)|^{2}\right)^{n} \log (2 /(1-|\varphi(z)|))}<\varepsilon, \\
\frac{\mu(z)\left|2 u^{\prime}(z) \varphi^{\prime}(z)+u(z) \varphi^{\prime \prime}(z)\right|}{\left(1-|\varphi(z)|^{2}\right)^{n+1} \log (2 /(1-|\varphi(z)|))}<\varepsilon, \\
\frac{\mu(z)\left|u(z)\left(\varphi^{\prime}(z)\right)^{2}\right|}{\left(1-|\varphi(z)|^{2}\right)^{n+2} \log (2 /(1-|\varphi(z)|))}<\varepsilon .
\end{gathered}
$$

From the boundedness of $\mathscr{D}_{\varphi, u}^{n}: \mathscr{B}_{\log } \rightarrow \mathscr{Z}_{\mu}$ by Theorem 5, we see that (23), (25), and (27) hold. Since $f_{k} \rightarrow 0$ uniformly on compact subsets of $\mathbb{D}$, Cauchy's estimate gives that $f_{k}^{(n)}$, $f_{k}^{(n+1)}$, and $f_{k}^{(n+2)}$ converge to 0 uniformly on compact subsets of $\mathbb{D}$; there exists a $K_{0} \in \mathbb{N}$ such that $k>K_{0}$ implies that

$$
\begin{aligned}
& \left|\left(\mathscr{D}_{\varphi, u}^{n} f_{k}\right)(0)\right|+\left|\left(\mathscr{D}_{\varphi, u}^{n} f_{k}\right)^{\prime}(0)\right| \\
& +\sup _{|\varphi(z)| \leq \rho} \mu(z)\left|\left(\mathscr{D}_{\varphi, u}^{n} f_{k}\right)^{\prime \prime}(z)\right| \\
& \leq|u(0)|\left|f_{k}^{(n)}(\varphi(0))\right|+\left|u^{\prime}(0)\right|\left|f_{k}^{(n)}(\varphi(0))\right| \\
& +|u(0)|\left|f_{k}^{(n+1)}(\varphi(0))\right|\left|\varphi^{\prime}(0)\right| \\
& +\sup _{|\varphi(z)| \leq \rho} \mu(z)\left|u^{\prime \prime}(z)\right|\left|f_{k}^{(n)}(\varphi(z))\right| \\
& +\sup \mu(z)\left|2 u^{\prime}(z) \varphi^{\prime}(z)+u(z) \varphi^{\prime \prime}(z)\right|\left|f_{k}^{(n+1)}(\varphi(z))\right| \\
& |\varphi(z)| \leq \rho \\
& +\sup _{|\varphi(z)| \leq \rho} \mu(z)\left|u(z)\left(\varphi^{\prime}(z)\right)^{2}\right|\left|f_{k}^{(n+2)}(\varphi(z))\right| \\
& \leq|u(0)|\left|f_{k}^{(n)}(\varphi(0))\right|+\left|u^{\prime}(0)\right|\left|f_{k}^{(n)}(\varphi(0))\right| \\
& +|u(0)|\left|\varphi^{\prime}(0)\right|\left|f_{k}^{(n+1)}(\varphi(0))\right| \\
& +K_{1} \sup _{|\varphi(z)| \leq \rho}\left|f_{k}^{(n)}(\varphi(z))\right|+K_{2} \sup _{|\varphi(z)| \leq \rho}\left|f_{k}^{(n+1)}(\varphi(z))\right| \\
& +K_{3} \sup _{|\varphi(z)| \leq \rho}\left|f_{k}^{(n+2)}(\varphi(z))\right|<C \varepsilon .
\end{aligned}
$$

From (52) and (53) and Lemma 1 we have

$$
\begin{aligned}
& \left\|\mathscr{D}_{\varphi, u}^{n} f_{k}\right\|_{\mathscr{X}_{\mu}} \\
& =\left|\left(\mathscr{D}_{\varphi, u}^{n} f_{k}\right)(0)\right|+\left|\left(\mathscr{D}_{\varphi, u}^{n} f_{k}\right)^{\prime}(0)\right|+\sup _{z \in \mathbb{D}} \mu(z)\left|\left(\mathscr{D}_{\varphi, u}^{n} f_{k}\right)^{\prime \prime}(z)\right| \\
& \leq \quad\left|\left(\mathscr{D}_{\varphi, u}^{n} f_{k}\right)(0)\right|+\left|\left(\mathscr{D}_{\varphi, u}^{n} f_{k}\right)^{\prime}(0)\right| \\
& \left.\quad+\sup _{|\varphi(z)| \leq \rho} \mu(z)\left|\left(\mathscr{D}_{\varphi, u}^{n} f_{k}\right)^{\prime \prime}(z)\right|\right) \\
& +\sup _{\rho<|\varphi(z)|<1} \mu(z)\left|\left(\mathscr{D}_{\varphi, u}^{n} f_{k}\right)^{\prime \prime}(z)\right| \\
& <C \varepsilon+\sup _{\rho<|\varphi(z)|<1} \frac{\mu^{\prime}\left(1-|\varphi(z)|^{2}\right)^{n} \log (2 /(1-|\varphi(z)|))}{(1 /(z) \mid} C \|_{\mathscr{B}_{\log }} \\
& \quad+\sup _{\rho<|\varphi(z)|<1} \frac{\mu(z)\left|2 u^{\prime}(z) \varphi^{\prime}(z)+u(z) \varphi^{\prime \prime}(z)\right|}{\left(1-|\varphi(z)|^{2}\right)^{n+1} \log (2 /(1-|\varphi(z)|))} C\|f\|_{\mathscr{B}_{\log }}
\end{aligned}
$$




$$
\begin{aligned}
& +\sup _{\rho<|\varphi(z)|<1} \frac{\mu(z)\left|u(z)\left(\varphi^{\prime}(z)\right)^{2}\right|}{\left(1-|\varphi(z)|^{2}\right)^{n+2} \log (2 /(1-|\varphi(z)|))} C\|f\|_{\mathscr{B}_{\log }} \\
& <4 C \varepsilon,
\end{aligned}
$$

when $K>K_{0}$. It follows that the operator $\mathscr{D}_{\varphi, u}^{n}: \mathscr{B}_{\log } \rightarrow \mathscr{Z}_{\mu}$ is compact.

(1) $\Rightarrow(2)$. It is obvious.

$(2) \Rightarrow$ (3). Assume that $\mathscr{D}_{\varphi, u}^{n}: \mathscr{B}_{\log , 0} \rightarrow \mathscr{Z}_{\mu}$ is compact. Then it is clear that $\mathscr{D}_{\varphi, u}^{n}: \mathscr{B}_{\log , 0} \rightarrow \mathscr{Z}_{\mu}$ is bounded. By Theorem 5 we get that $\mathscr{D}_{\varphi, u}^{n}: \mathscr{B}_{\log } \rightarrow \mathscr{Z}_{\mu}$ is bounded. Let $\left\{z_{k}\right\}$ be a sequence in $\mathbb{D}$ such that $\left|\varphi\left(z_{k}\right)\right| \rightarrow 1$ as $k \rightarrow \infty$. We can use the test functions

$$
\begin{aligned}
f_{k}(z)= & f_{z_{k}}(z) \\
= & (n+2)(n+3) \frac{1-\left|\varphi\left(z_{k}\right)\right|^{2}}{\left(1-z \overline{\varphi\left(z_{k}\right)}\right) \log \left(2 /\left(1-\left|\varphi\left(z_{k}\right)\right|\right)\right)} \\
& -2(n+3) \frac{\left(1-\left|\varphi\left(z_{k}\right)\right|^{2}\right)^{2}}{\left(1-z \overline{\varphi\left(z_{k}\right)}\right)^{2} \log \left(2 /\left(1-\left|\varphi\left(z_{k}\right)\right|\right)\right)} \\
& +2 \frac{\left(1-\left|\varphi\left(z_{k}\right)\right|^{2}\right)^{3}}{\left(1-z \overline{\varphi\left(z_{k}\right)}\right)^{3} \log \left(2 /\left(1-\left|\varphi\left(z_{k}\right)\right|\right)\right)}
\end{aligned}
$$

Note that

$$
\begin{aligned}
\left|f_{k}(z)\right| \leq & \left|\frac{(n+2)(n+3)\left(1-\left|\varphi\left(z_{k}\right)\right|^{2}\right)}{\left(1-\overline{\varphi\left(z_{k}\right)}\right) \log \left(2 /\left(1-\left|\varphi\left(z_{k}\right)\right|\right)\right)}\right| \\
& +\left|\frac{2(n+3)\left(1-\left|\varphi\left(z_{k}\right)\right|^{2}\right)^{2}}{\left(1-\overline{\varphi_{(}\left(z_{k}\right)}\right)^{2} \log \left(2 /\left(1-\left|\varphi\left(z_{k}\right)\right|\right)\right)}\right| \\
& +\left|\frac{2\left(1-\left|\varphi\left(z_{k}\right)\right|^{2}\right)^{3}}{\left(1-z \overline{\left(z_{k}\right)}\right)^{3} \log \left(2 /\left(1-\left|\varphi\left(z_{k}\right)\right|\right)\right)}\right| \\
\leq & \frac{(n+2)(n+3)\left(1+\left|\varphi\left(z_{k}\right)\right|\right)\left(1-\left|\varphi\left(z_{k}\right)\right|\right)}{\left(1-\left|\varphi\left(z_{k}\right)\right|\right) \log \left(2 /\left(1-\left|\varphi\left(z_{k}\right)\right|\right)\right)} \\
& +\frac{2(n+3)\left(1+\left|\varphi\left(z_{k}\right)\right|\right)^{2}\left(1-\left|\varphi\left(z_{k}\right)\right|\right)^{2}}{\left(1-\left|\varphi\left(z_{k}\right)\right|\right)^{2} \log \left(2 /\left(1-\left|\varphi\left(z_{k}\right)\right|\right)\right)} \\
\leq & \frac{2\left(1+\left|\varphi\left(z_{k}\right)\right|\right)^{3}\left(1-\left|\varphi\left(z_{k}\right)\right|\right)^{3}}{\log \left(2 /\left(1-\left|\varphi\left(z_{k}\right)\right|\right)\right)}+\frac{8(n+3)}{\log \left(2 /\left(1-\left|\varphi\left(z_{k}\right)\right|\right)\right)} \\
& \frac{\left.2\left(z_{k}\right) \mid\right)^{3} \log \left(2 /\left(1-\left|\varphi\left(z_{k}\right)\right|\right)\right)}{(n+3)}
\end{aligned}
$$

$$
\begin{aligned}
& +\frac{16}{\log \left(2 /\left(1-\left|\varphi\left(z_{k}\right)\right|\right)\right)} \\
= & \frac{2 n^{2}+18 n+52}{\log \left(2 /\left(1-\left|\varphi\left(z_{k}\right)\right|\right)\right)} \longrightarrow 0, \quad(k \rightarrow \infty),
\end{aligned}
$$

for $|z|<1$. We see that $f_{k}$ converges to 0 uniformly on $\mathbb{D}$; hence, $f_{k}$ converges to 0 uniformly on compact subsets of $\mathbb{D}$ and from (30) and (33) we have $\sup _{k \in \mathbb{N}}\left\|f_{k}\right\|_{\mathscr{B}_{\log }} \leq$ $C, f_{k} \in \mathscr{B}_{\log , 0}$. Then $f_{k}$ is a bounded sequence in $\mathscr{B}_{\log , 0}$ which converges to 0 uniformly on compact subsets of $\mathbb{D}$. By Lemma 3, we have

$$
\lim _{k \rightarrow \infty}\left\|\mathscr{D}_{\varphi, u}^{n} f_{k}\right\|_{\mathscr{E}_{\mu}}=0
$$

Note that

$$
\begin{gathered}
f_{k}^{(n+1)}\left(\varphi\left(z_{k}\right)\right)=f_{k}^{(n+2)}\left(\varphi\left(z_{k}\right)\right)=0 \\
f_{k}^{(n)}\left(\varphi\left(z_{k}\right)\right)=2 \cdot n ! \frac{\left(\overline{\varphi\left(z_{k}\right)}\right)^{n}}{\left(1-\left|\varphi\left(z_{k}\right)\right|^{2}\right)^{n} \log \left(2 /\left(1-\left|\varphi\left(z_{k}\right)\right|\right)\right)} .
\end{gathered}
$$

From (33) and using the compactness of $\mathscr{D}_{\varphi, u}^{n}: \mathscr{B}_{\log , 0} \rightarrow \mathscr{Z}_{\mu}$ we obtain

$$
\begin{aligned}
2 \cdot n ! & \frac{\mu\left(z_{k}\right)\left|u^{\prime \prime}\left(z_{k}\right)\right|\left|\overline{\varphi\left(z_{k}\right)}\right|^{n}}{\left(1-\left|\varphi\left(z_{k}\right)\right|^{2}\right)^{n} \log \left(2 /\left(1-\left|\varphi\left(z_{k}\right)\right|\right)\right)} \\
& \leq\left\|\mathscr{D}_{\varphi, u}^{n} f_{k}\right\|_{\mathscr{F}_{\mu}} \longrightarrow 0, \quad \text { as } k \longrightarrow \infty .
\end{aligned}
$$

From (59) and $\left|\varphi\left(z_{k}\right)\right| \rightarrow 1$, it follows that

$$
\lim _{k \rightarrow \infty} \frac{\mu\left(z_{k}\right)\left|u^{\prime \prime}\left(z_{k}\right)\right|}{\left(1-\left|\varphi\left(z_{k}\right)\right|^{2}\right)^{n} \log \left(2 /\left(1-\left|\varphi\left(z_{k}\right)\right|\right)\right)}=0
$$

and consequently (48) holds.

Next, let

$$
\begin{aligned}
g_{k}(z)= & g_{z_{k}}(z) \\
= & (n+1)(n+3) \frac{1-\left|\varphi\left(z_{k}\right)\right|^{2}}{\left(1-z \overline{\varphi\left(z_{k}\right)}\right) \log \left(2 /\left(1-\left|\varphi\left(z_{k}\right)\right|\right)\right)} \\
& -(2 n+5) \frac{\left(1-\left|\varphi\left(z_{k}\right)\right|^{2}\right)^{2}}{\left(1-z \overline{\varphi\left(z_{k}\right)}\right)^{2} \log \left(2 /\left(1-\left|\varphi\left(z_{k}\right)\right|\right)\right)} \\
& +2 \frac{\left(1-\left|\varphi\left(z_{k}\right)\right|^{2}\right)^{3}}{\left(1-z \overline{\varphi\left(z_{k}\right)}\right)^{3} \log \left(2 /\left(1-\left|\varphi\left(z_{k}\right)\right|\right)\right)} .
\end{aligned}
$$

By a direct calculation, we obtain that $g_{k}$ converges to 0 uniformly on compact subsets of $\mathbb{D}, g_{k} \in \mathscr{B}_{\log , 0}$, and $\sup _{k \in \mathbb{N}}\left\|g_{k}\right\|_{\mathscr{B}_{\log }} \leq C$. By Lemma 3, we have

$$
\lim _{k \rightarrow \infty}\left\|\mathscr{D}_{\varphi, u}^{n} g_{k}\right\|_{\mathscr{I}_{\mu}}=0
$$


Note that

$$
\begin{gathered}
g_{k}^{(n+1)}\left(\varphi\left(z_{k}\right)\right) \\
=-(n+1) ! \frac{\left(\overline{\varphi\left(z_{k}\right)}\right)^{n+1}}{\left(1-\left|\varphi\left(z_{k}\right)\right|^{2}\right)^{n+1} \log \left(2 /\left(1-\left|\varphi\left(z_{k}\right)\right|\right)\right)}, \\
g_{k}^{(n)}\left(\varphi\left(z_{k}\right)\right)=g_{\omega}^{(n+2)}\left(\varphi\left(z_{k}\right)\right)=0 .
\end{gathered}
$$

From (39) and using the compactness of $\mathscr{D}_{\varphi, u}^{n}: \mathscr{B}_{\log , 0} \rightarrow \mathscr{Z}_{\mu}$ we obtain

$$
\begin{gathered}
(n+1) ! \frac{\mu\left(z_{k}\right)\left|2 u^{\prime}\left(z_{k}\right) \varphi^{\prime}\left(z_{k}\right)+u\left(z_{k}\right) \varphi^{\prime \prime}\left(z_{k}\right)\right|\left|\overline{\varphi\left(z_{k}\right)}\right|^{n+1}}{\left(1-\left|\varphi\left(z_{k}\right)\right|^{2}\right)^{n+1} \log \left(2 /\left(1-\left|\varphi\left(z_{k}\right)\right|\right)\right)} \\
\leq\left\|\mathscr{D}_{\varphi, u}^{n} g_{k}\right\|_{\mathscr{X}_{\mu}} \longrightarrow 0, \quad \text { as } k \longrightarrow \infty .
\end{gathered}
$$

From (64) and $\left|\varphi\left(z_{k}\right)\right| \rightarrow 1$, we have

$$
\lim _{k \rightarrow \infty} \frac{\mu\left(z_{k}\right)\left|2 u^{\prime}\left(z_{k}\right) \varphi^{\prime}\left(z_{k}\right)+u\left(z_{k}\right) \varphi^{\prime \prime}\left(z_{k}\right)\right|}{\left(1-\left|\varphi\left(z_{k}\right)\right|^{2}\right)^{n+1} \log \left(2 /\left(1-\left|\varphi\left(z_{k}\right)\right|\right)\right)}=0 ;
$$

it implies that (49) holds.

In order to prove $(50)$, choose

$$
\begin{aligned}
h_{k}(z)= & h_{z_{k}}(z) \\
= & (n+1)(n+2) \frac{1-\left|\varphi\left(z_{k}\right)\right|^{2}}{\left(1-z \overline{\varphi\left(z_{k}\right)}\right) \log \left(2 /\left(1-\left|\varphi\left(z_{k}\right)\right|\right)\right)} \\
& -2(n+2) \frac{\left(1-\left|\varphi\left(z_{k}\right)\right|^{2}\right)^{2}}{\left(1-z \overline{\varphi\left(z_{k}\right)}\right)^{2} \log \left(2 /\left(1-\left|\varphi\left(z_{k}\right)\right|\right)\right)} \\
& +2 \frac{\left(1-\left|\varphi\left(z_{k}\right)\right|^{2}\right)^{3}}{\left(1-z \overline{\varphi\left(z_{k}\right)}\right)^{3} \log \left(2 /\left(1-\left|\varphi\left(z_{k}\right)\right|\right)\right)} .
\end{aligned}
$$

By a direct calculation, we may easily prove that $h_{k}$ converges to 0 uniformly on compact subsets of $\mathbb{D}, h_{k} \in \mathscr{B}_{\log , 0}$, and $\sup _{k \in \mathbb{N}}\left\|h_{k}\right\|_{\mathscr{B}_{\log }} \leq$ C. By Lemma 3, we have

$$
\lim _{k \rightarrow \infty}\left\|\mathscr{D}_{\varphi, u}^{n} h_{k}\right\|_{\mathscr{X}_{\mu}}=0
$$

Note that

$$
\begin{gathered}
h_{k}^{(n+2)}\left(\varphi\left(z_{k}\right)\right) \\
=2 \cdot(n+2) ! \frac{\left(\overline{\varphi\left(z_{k}\right)}\right)^{n+2}}{\left(1-\left|\varphi\left(z_{k}\right)\right|^{2}\right)^{n+2} \log \left(2 /\left(1-\left|\varphi\left(z_{k}\right)\right|\right)\right)} \\
h_{k}^{(n)}\left(\varphi\left(z_{k}\right)\right)=h_{k}^{(n+1)}\left(\varphi\left(z_{k}\right)\right)=0 .
\end{gathered}
$$

From (45) and using the compactness of $\mathscr{D}_{\varphi, u}^{n}: \mathscr{B}_{\log , 0} \rightarrow \mathscr{Z}_{\mu}$ we obtain

$$
\begin{gathered}
2 \cdot(n+2) ! \frac{\mu\left(z_{k}\right)\left|u\left(z_{k}\right)\left(\varphi^{\prime}\left(z_{k}\right)\right)^{2}\right|\left|\overline{\varphi\left(z_{k}\right)}\right|^{n+2}}{\left(1-\left|\varphi\left(z_{k}\right)\right|^{2}\right)^{n+2} \log \left(2 /\left(1-\left|\varphi\left(z_{k}\right)\right|\right)\right)} \\
\leq\left\|\mathscr{D}_{\varphi, u}^{n} h_{k}\right\|_{\mathscr{X}_{\mu}} \longrightarrow 0, \quad \text { as } k \longrightarrow \infty .
\end{gathered}
$$

From (69) and $\left|\varphi\left(z_{k}\right)\right| \rightarrow 1$, it follows that

$$
\lim _{k \rightarrow \infty} \frac{\mu\left(z_{k}\right)\left|u\left(z_{k}\right)\left(\varphi^{\prime}\left(z_{k}\right)\right)^{2}\right|}{\left(1-\left|\varphi\left(z_{k}\right)\right|^{2}\right)^{n+2} \log \left(2 /\left(1-\left|\varphi\left(z_{k}\right)\right|\right)\right)}=0
$$

and consequently (50) holds, finishing the proof of the theorem.

Theorem 7. Let $u \in H(\mathbb{D})$, and let $n$ be a nonnegative integer, $\varphi$ a holomorphic self-map of $\mathbb{D}$, and $\mu$ a weight. Then $\mathscr{D}_{\varphi, u}^{n}: \mathscr{B}_{\log } \rightarrow \mathscr{Z}_{\mu, 0}$ is a bounded operator provided that the following conditions are satisfied:

$$
\begin{gathered}
\lim _{|z| \rightarrow 1} \frac{\mu(z)\left|u^{\prime \prime}(z)\right|}{\left(1-|\varphi(z)|^{2}\right)^{n} \log (2 /(1-|\varphi(z)|))}=0, \\
\lim _{|z| \rightarrow 1} \frac{\mu(z)\left|2 u^{\prime}(z) \varphi^{\prime}(z)+u(z) \varphi^{\prime \prime}(z)\right|}{\left(1-|\varphi(z)|^{2}\right)^{n+1} \log (2 /(1-|\varphi(z)|))}=0, \\
\lim _{|z| \rightarrow 1} \frac{\mu(z)\left|u(z)\left(\varphi^{\prime}(z)\right)^{2}\right|}{\left(1-|\varphi(z)|^{2}\right)^{n+2} \log (2 /(1-|\varphi(z)|))}=0 .
\end{gathered}
$$

Proof. Suppose that (71), (72), and (73) hold. It is clear that (17), (18), and (19) hold. By Theorem 5 we have that $\mathscr{D}_{\varphi, u}^{n}$ : $\mathscr{B}_{\log } \rightarrow \mathscr{Z}_{\mu}$ is bounded. In order to prove $\mathscr{D}_{\varphi, u}^{n}: \mathscr{B}_{\log } \rightarrow$ $\mathscr{Z}_{\mu, 0}$ is bounded, it is enough to show that, for any $f \in \mathscr{B}_{\log }$, $\mathscr{D}_{\varphi, u}^{n} f \in \mathscr{Z}_{\mu, 0}$. Using (71), (72), and (73) we have that, for any $\varepsilon>0$, there is a constant $0<\eta<1$, such that $\eta<|z|<1$ implies

$$
\begin{gathered}
\frac{\mu(z)\left|u^{\prime \prime}(z)\right|}{\left(1-|\varphi(z)|^{2}\right)^{n} \log (2 /(1-|\varphi(z)|))}<\varepsilon, \\
\frac{\mu(z)\left|2 u^{\prime}(z) \varphi^{\prime}(z)+u(z) \varphi^{\prime \prime}(z)\right|}{\left(1-|\varphi(z)|^{2}\right)^{n+1} \log (2 /(1-|\varphi(z)|))}<\varepsilon, \\
\frac{\mu(z)\left|u(z)\left(\varphi^{\prime}(z)\right)^{2}\right|}{\left(1-|\varphi(z)|^{2}\right)^{n+2} \log (2 /(1-|\varphi(z)|))}<\varepsilon .
\end{gathered}
$$


Then, for any $f \in \mathscr{B}_{\text {log }}$, from Lemma 1 we obtain that

$$
\begin{aligned}
& \mu(z)\left|\left(\mathscr{D}_{\varphi, u}^{n} f\right)^{\prime \prime}(z)\right| \\
& =\mu(z) \mid u^{\prime \prime}(z) f^{(n)}(\varphi(z)) \\
& +\left(2 u^{\prime}(z) \varphi^{\prime}(z)+u(z) \varphi^{\prime \prime}(z)\right) f^{(n+1)}(\varphi(z)) \\
& +u(z)\left(\varphi^{\prime}(z)\right)^{2} f^{(n+2)}(\varphi(z)) \\
& \leq \mu(z)\left|u^{\prime \prime}(z)\right|\left|f^{(n)}(\varphi(z))\right| \\
& +\mu(z)\left|2 u^{\prime}(z) \varphi^{\prime}(z)+u(z) \varphi^{\prime \prime}(z)\right|\left|f^{(n+1)}(\varphi(z))\right| \\
& +\mu(z)\left|u(z)\left(\varphi^{\prime}(z)\right)^{2}\right|\left|f^{(n+2)}(\varphi(z))\right| \\
& \leq C\|f\|_{\mathscr{B}_{\log }} \frac{\mu(z)\left|u^{\prime \prime}(z)\right|}{\left(1-|\varphi(z)|^{2}\right)^{n} \log (2 /(1-|\varphi(z)|))} \\
& +C\|f\|_{\mathscr{B}_{\log }} \frac{\mu(z)\left|2 u^{\prime}(z) \varphi^{\prime}(z)+u(z) \varphi^{\prime \prime}(z)\right|}{\left(1-|\varphi(z)|^{2}\right)^{n+1} \log (2 /(1-|\varphi(z)|))} \\
& +C\|f\|_{\mathscr{B}_{\log }} \frac{\mu(z)\left|u(z)\left(\varphi^{\prime}(z)\right)^{2}\right|}{\left(1-|\varphi(z)|^{2}\right)^{n+2} \log (2 /(1-|\varphi(z)|))} \\
& <3 C\|f\|_{\mathscr{B}_{\log }} \mathcal{E}
\end{aligned}
$$

when $\eta<|z|<1$. Hence $\mathscr{D}_{\varphi, u}^{n} f \in \mathscr{Z}_{\mu, 0}$ for all $f \in \mathscr{B}_{\text {log }}$, completing the proof of the theorem.

Theorem 8. Let $u \in H(\mathbb{D})$, and let $n$ be a nonnegative integer, $\varphi$ a holomorphic self-map of $\mathbb{D}$, and $\mu$ a weight. If $\mathscr{D}_{\varphi, u}^{n}$ : $\mathscr{B}_{\log } \rightarrow \mathscr{E}_{\mu, 0}$ is a bounded operator, then (17), (18), and (19) hold and the following conditions are satisfied:

$$
\begin{gathered}
\lim _{|z| \rightarrow 1} \mu(z)\left|u^{\prime \prime}(z)\right|=0, \\
\lim _{|z| \rightarrow 1} \mu(z)\left|2 u^{\prime}(z) \varphi^{\prime}(z)+u(z) \varphi^{\prime \prime}(z)\right|=0, \\
\lim _{|z| \rightarrow 1} \mu(z)\left|u(z)\left(\varphi^{\prime}(z)\right)^{2}\right|=0 .
\end{gathered}
$$

Proof. Assume that $\mathscr{D}_{\varphi, u}^{n}: \mathscr{B}_{\log } \rightarrow \mathscr{Z}_{\mu, 0}$ is bounded; it is clear that $\mathscr{D}_{\varphi, u}^{n}: \mathscr{B}_{\log } \rightarrow \mathscr{Z}_{\mu}$ is bounded. By Theorem 5 we have that (17), (18), and (19) hold. On the other hand, for all $f \in \mathscr{B}_{\log }, \mathscr{D}_{\varphi, u}^{n} f \in \mathscr{Z}_{\mu, 0}$. Take $f(z)=z^{n} / n ! \in \mathscr{B}_{\text {log }}$; we have that

$$
\lim _{|z| \rightarrow 1} \mu(z)\left|u^{\prime \prime}(z)\right|=0
$$

then (76) holds. Let $f(z)=z^{n+1} /(n+1) ! \in \mathscr{B}_{\log }$; we have that

$$
\lim _{|z| \rightarrow 1} \mu(z)\left|u^{\prime \prime}(z) \varphi(z)+2 u^{\prime}(z) \varphi^{\prime}(z)+u(z) \varphi^{\prime \prime}(z)\right|=0 .
$$

By (80), (76), and the boundedness of the function $\varphi(z)$, we get

$$
\lim _{|z| \rightarrow 1} \mu(z)\left|2 u^{\prime}(z) \varphi^{\prime}(z)+u(z) \varphi^{\prime \prime}(z)\right|=0 .
$$

Hence, (77) holds. In the same way, let $f(z)=z^{n+2} /(n+2) ! \epsilon$ $\mathscr{B}_{\text {log }}$; we have that

$$
\begin{aligned}
\lim _{|z| \rightarrow 1} \mu(z) \mid u^{\prime \prime}(z)(\varphi(z))^{2} & \\
& +2\left(2 u^{\prime}(z) \varphi^{\prime}(z)+u(z) \varphi^{\prime \prime}(z)\right) \varphi(z) \\
& +2 u(z)\left(\varphi^{\prime}(z)\right)^{2} \mid=0 .
\end{aligned}
$$

By (76), (77), (82), and the boundedness of the function $\varphi(z)$, we have that

$$
\lim _{|z| \rightarrow 1} \mu(z)\left|u(z)\left(\varphi^{\prime}(z)\right)^{2}\right|=0 .
$$

That is, (78) holds. The proof is completed.

Theorem 9. Let $u \in H(\mathbb{D})$, and let $n$ be a nonnegative integer, $\varphi$ a holomorphic self-map of $\mathbb{D}$, and $\mu$ a weight. Then $\mathscr{D}_{\varphi, u}^{n}$ : $\mathscr{B}_{\log , 0} \rightarrow \mathscr{Z}_{\mu, 0}$ is a bounded operator if and only if $\mathscr{D}_{\varphi, u}^{n}:$ $\mathscr{B}_{\log , 0} \rightarrow \mathscr{Z}_{\mu}$ is a bounded operator and (76), (77), and (78) hold.

Proof. Assume that $\mathscr{D}_{\varphi, u}^{n}: \mathscr{B}_{\log , 0} \rightarrow \mathscr{Z}_{\mu, 0}$ is a bounded operator; it is clear that $\mathscr{D}_{\varphi, u}^{n}: \mathscr{B}_{\log , 0} \rightarrow \mathscr{Z}_{\mu}$ is a bounded operator. On the other hand, for all $f \in \mathscr{B}_{\log , 0}, \mathscr{D}_{\varphi, u}^{n} f \in \mathscr{Z}_{\mu, 0}$. Taking $f(z)=z^{n} / n ! \in \mathscr{B}_{\log , 0}$, we have that

$$
\lim _{|z| \rightarrow 1} \mu(z)\left|u^{\prime \prime}(z)\right|=0
$$

then (76) holds. Let $f(z)=z^{n+1} /(n+1) ! \in \mathscr{B}_{\log , 0}$; we have that

$$
\lim _{|z| \rightarrow 1} \mu(z)\left|u^{\prime \prime}(z) \varphi(z)+2 u^{\prime}(z) \varphi^{\prime}(z)+u(z) \varphi^{\prime \prime}(z)\right|=0 .
$$

By (85), (76), and the boundedness of the function $\varphi(z)$, we get

$$
\lim _{|z| \rightarrow 1} \mu(z)\left|2 u^{\prime}(z) \varphi^{\prime}(z)+u(z) \varphi^{\prime \prime}(z)\right|=0 .
$$

Hence, (77) holds. In the same way, take $f(z)=z^{n+2} /(n+2) ! \epsilon$ $\mathscr{B}_{\log , 0}$; we have that

$$
\begin{aligned}
\lim _{|z| \rightarrow 1} \mu(z) \mid u^{\prime \prime}(z)(\varphi(z))^{2} & \\
& +2\left(2 u^{\prime}(z) \varphi^{\prime}(z)+u(z) \varphi^{\prime \prime}(z)\right) \varphi(z) \\
& +2 u(z)\left(\varphi^{\prime}(z)\right)^{2} \mid=0 .
\end{aligned}
$$


By (76), (77), (87), and the boundedness of the function $\varphi(z)$, we have that

$$
\lim _{|z| \rightarrow 1} \mu(z)\left|u(z)\left(\varphi^{\prime}(z)\right)^{2}\right|=0 .
$$

That is, (78) holds.

Conversely, suppose that $\mathscr{D}_{\varphi, u}^{n}: \mathscr{B}_{\log , 0} \rightarrow \mathscr{Z}_{\mu}$ is a bounded operator and (76), (77), and (78) hold. For each polynomial $p(z)$ we get that

$$
\begin{aligned}
& \mu(z)\left|\left(\mathscr{D}_{\varphi, u}^{n} p\right)^{\prime \prime}(z)\right| \\
& =\mu(z) \mid u^{\prime \prime}(z) p^{(n)}(\varphi(z)) \\
& \quad+\left(2 u^{\prime}(z) \varphi^{\prime}(z)+u(z) \varphi^{\prime \prime}(z)\right) p^{(n+1)}(\varphi(z)) \\
& \quad+u(z)\left(\varphi^{\prime}(z)\right)^{2} p^{(n+2)}(\varphi(z)) \mid \\
& \leq \mu(z)\left|u^{\prime \prime}(z)\right|\left|p^{(n)}(\varphi(z))\right| \\
& +\mu(z)\left|2 u^{\prime}(z) \varphi^{\prime}(z)+u(z) \varphi^{\prime \prime}(z)\right|\left|p^{(n+1)}(\varphi(z))\right| \\
& \quad+\mu(z)\left|u(z)\left(\varphi^{\prime}(z)\right)^{2}\right|\left|p^{(n+2)}(\varphi(z))\right| \\
& \leq \mu(z)\left|u^{\prime \prime}(z)\right|\left\|p^{(n)}\right\|_{\infty} \\
& \quad+\mu(z)\left|2 u^{\prime}(z) \varphi^{\prime}(z)+u(z) \varphi^{\prime \prime}(z)\right|\left\|p^{(n+1)}\right\|_{\infty} \\
& +\mu(z)\left|u(z)\left(\varphi^{\prime}(z)\right)^{2}\right|\left\|p^{(n+2)}\right\|_{\infty} \longrightarrow
\end{aligned}
$$$$
\text { (as }|z| \longrightarrow 1) \text {. }
$$

Hence, $\mathscr{D}_{\varphi, u}^{n} p \in \mathscr{Z}_{\mu, 0}$. On the other hand, since polynomials are dense in $\mathscr{B}_{\log , 0}$, thus, for each $f \in \mathscr{B}_{\log , 0}$, there is a sequence of polynomials $\left\{p_{k}\right\}_{k \in \mathbb{N}}$ such that

$$
\lim _{k \rightarrow \infty}\left\|p_{k}-f\right\|_{\mathscr{B}_{\log }}=0
$$

Since $\mathscr{D}_{\varphi, u}^{n}: \mathscr{B}_{\log , 0} \rightarrow \mathscr{Z}_{\mu}$ is a bounded operator, by Theorem 5 we have $\mathscr{D}_{\varphi, u}^{n}: \mathscr{B}_{\log } \rightarrow \mathscr{Z}_{\mu}$ is a bounded operator. Since

$$
\left\|\mathscr{D}_{\varphi, u}^{n} p_{k}-\mathscr{D}_{\varphi, u}^{n} f\right\|_{\mathscr{Z}_{\mu}} \leq\left\|\mathscr{D}_{\varphi, u}^{n}\right\|\left\|p_{k}-f\right\|_{\mathscr{B}_{\log }}
$$

and $\mathscr{Z}_{\mu, 0}$ is the closed subset of $\mathscr{Z}_{\mu}$, we see that $\mathscr{D}_{\varphi, u}^{n} f \in \mathscr{Z}_{\mu, 0}$; thus $\mathscr{D}_{\varphi, u}^{n}: \mathscr{B}_{\log , 0} \rightarrow \mathscr{Z}_{\mu, 0}$ is a bounded operator. The proof is completed.

Theorem 10. Let $u \in H(\mathbb{D})$, and let $n$ be a nonnegative integer, $\varphi$ a holomorphic self-map of $\mathbb{D}$, and $\mu$ a weight. Then the following statements are equivalent:

(1) $\mathscr{D}_{\varphi, u}^{n}: \mathscr{B}_{\log } \rightarrow \mathscr{Z}_{\mu, 0}$ is compact;

(2) $\mathscr{D}_{\varphi, u}^{n}: \mathscr{B}_{\log , 0} \rightarrow \mathscr{Z}_{\mu, 0}$ is compact;

(3) (71), (72), and (73) hold.
Proof. (3) $\Rightarrow$ (1). Suppose that (71) (72), and (73) hold. By Theorem 7 we know that $\mathscr{D}_{\varphi, u}^{n}: \mathscr{B}_{\log } \rightarrow \mathscr{Z}_{\mu, 0}$ is bounded. Taking the supremum in inequality (20) over all $f \in \mathscr{B}_{\log }$ such that $\|f\|_{\mathscr{B}_{\log }} \leq 1$ and letting $|z| \rightarrow 1$ yields

$$
\lim _{|z| \rightarrow 1} \sup _{\|f\|_{\mathscr{B}_{\log }} \leq 1} \mu(z)\left|\left(\mathscr{D}_{\varphi, u}^{n} f\right)^{\prime \prime}(z)\right|=0 .
$$

Hence, by Lemma 4 , we see that the operator $\mathscr{D}_{\varphi, u}^{n}: \mathscr{B}_{\log } \rightarrow$ $\mathscr{Z}_{\mu, 0}$ is compact.

$(1) \Rightarrow(2)$. This implication is clear.

$(2) \Rightarrow$ (3). Assume that $\mathscr{D}_{\varphi, u}^{n}: \mathscr{B}_{\log , 0} \rightarrow \mathscr{Z}_{\mu, 0}$ is compact. Firstly, it is obvious $\mathscr{D}_{\varphi, u}^{n}: \mathscr{B}_{\log , 0} \rightarrow \mathscr{X}_{\mu, 0}$ is bounded. By Theorem 9 we have that (76), (77), and (78) hold. On the other hand, we have that $\mathscr{D}_{\varphi, u}^{n}: \mathscr{B}_{\log , 0} \rightarrow \mathscr{Z}_{\mu}$ is compact. By Theorem 6 we have that (48), (49), and (50) hold. We prove that (76) and (48) imply (71). The proof of (72) and (73) is similar; hence, it will be omitted. From (48), it follows that, for every $\varepsilon>0$, there exists $\delta \in(0,1)$ such that

$$
\frac{\mu(z)\left|u^{\prime \prime}(z)\right|}{\left(1-|\varphi(z)|^{2}\right)^{n} \log (2 /(1-|\varphi(z)|))}<\varepsilon,
$$

when $\delta<|\varphi(z)|<1$. Using (76) we see that there exists $\tau \in$ $(0,1)$ such that

$$
\mu(z)\left|u^{\prime \prime}(z)\right| \leq \varepsilon \inf _{t \in[0, \delta]}\left(1-t^{2}\right)^{n} \log 2,
$$

when $\tau<|z|<1$. Therefore when $\tau<|z|<1$ and $\delta<$ $|\varphi(z)|<1$, by (93), we have

$$
\frac{\mu(z)\left|u^{\prime \prime}(z)\right|}{\left(1-|\varphi(z)|^{2}\right)^{n} \log (2 /(1-|\varphi(z)|))}<\varepsilon .
$$

On the other hand, when $\tau<|z|<1$ and $|\varphi(z)| \leq \delta$, by (94), we obtain

$$
\begin{gathered}
\frac{\mu(z)\left|u^{\prime \prime}(z)\right|}{\left(1-|\varphi(z)|^{2}\right)^{n} \log (2 /(1-|\varphi(z)|))} \\
\leq \frac{\mu(z)\left|u^{\prime \prime}(z)\right|}{\inf _{t \in[0, \delta]}\left(1-t^{2}\right)^{n} \log 2}<\varepsilon .
\end{gathered}
$$

From (95) and (96) we have

$$
\lim _{|z| \rightarrow 1} \frac{\mu(z)\left|u^{\prime \prime}(z)\right|}{\left(1-|\varphi(z)|^{2}\right)^{n} \log (2 /(1-|\varphi(z)|))}=0 ;
$$

we obtain that (71) holds, as desired. The proof is completed.

\section{Conflict of Interests}

The authors declare that there is no conflict of interests regarding the publication of this paper. 


\section{Acknowledgments}

The authors thank the referees for their thoughtful comments and helpful suggestions. This research was supported by the Natural Science Foundation of China (11171285) and the Priority Academic Program Development of Jiangsu Higher Education Institutions.

\section{References}

[1] K. R. M. Attele, "Toeplitz and Hankel operators on Bergman one space," Hokkaido Mathematical Journal, vol. 21, no. 2, pp. 279-293, 1992.

[2] S. Ye, "Multipliers and cyclic vectors on the weighted Bloch space," Mathematical Journal of Okayama University, vol. 48, pp. 135-143, 2006.

[3] P. Galanopoulos, "On $B_{\log }$ to $Q_{\log }^{p}$ pullbacks," Journal of Mathematical Analysis and Applications, vol. 337, no. 1, pp. 712-725, 2008.

[4] S. L. Ye, "A weighted composition operator between different weighted Bloch-type spaces," Acta Mathematica Sinica, vol. 50, no. 4, pp. 927-942, 2007 (Chinese).

[5] S. Li, "Weighted composition operators from Bergman spaces into weighted Bloch spaces," Communications of the Korean Mathematical Society, vol. 20, no. 1, pp. 63-70, 2005.

[6] S. Ye, "Weighted composition operators from $F(p, q, s)$ into logarithmic Bloch space," Journal of the Korean Mathematical Society, vol. 45, no. 4, pp. 977-991, 2008.

[7] F. Colonna and S. Li, "Weighted composition operators from Hardy spaces into logarithmic Bloch spaces," Journal of Function Spaces and Applications, vol. 2012, Article ID 454820, 20 pages, 2012.

[8] A. N. Petrov, "Reverse estimates in logarithmic Bloch spaces," Archiv der Mathematik, vol. 100, no. 6, pp. 551-560, 2013.

[9] R. E. Castillo, D. D. Clahane, J. F. Farías López, and J. C. Ramos Fernández, "Composition operators from logarithmic Bloch spaces to weighted Bloch spaces," Applied Mathematics and Computation, vol. 219, no. 12, pp. 6692-6706, 2013.

[10] A. J. García Ortiz and J. C. Ramos-Fernández, "Composition operators from logarithmic Bloch spaces to Bloch-type spaces," Georgian Mathematical Journal, vol. 20, no. 4, pp. 671-686, 2013.

[11] S. Li and S. Stević, "Volterra-type operators on Zygmund spaces," Journal of Inequalities and Applications, vol. 2007, Article ID 32124, 10 pages, 2007.

[12] S. Stević, "Weighted differentiation composition operators from mixed-norm spaces to weighted-type spaces," Applied Mathematics and Computation, vol. 211, no. 1, pp. 222-233, 2009.

[13] X. Zhu, "Products of differentiation, composition and multiplication from Bergman type spaces to Bers type spaces," Integral Transforms and Special Functions, vol. 18, no. 3-4, pp. 223-231, 2007.

[14] S. Li and S. Stević, "Weighted composition operators between $H^{\infty}$ and $\alpha$-Bloch spaces in the unit ball," Taiwanese Journal of Mathematics, vol. 12, no. 7, pp. 1625-1639, 2008.

[15] S. Stević, "Norm of weighted composition operators from Bloch space to $H_{\mu}^{\infty}$ on the unit ball," Ars Combinatoria, vol. 88, pp. $125-127,2008$.

[16] S. Stević, "Norm and essential norm of composition followed by differentiation from $\alpha$-Bloch spaces to $H_{\mu}^{\infty}$," Applied Mathematics and Computation, vol. 207, no. 1, pp. 225-229, 2009.
[17] R. A. Hibschweiler and N. Portnoy, "Composition followed by differentiation between Bergman and Hardy spaces," The Rocky Mountain Journal of Mathematics, vol. 35, no. 3, pp. 843-855, 2005.

[18] Y. Liu and Y. Yu, "Composition followed by differentiation between $H^{\infty}$ and Zygmund spaces," Complex Analysis and Operator Theory, vol. 6, no. 1, pp. 121-137, 2012.

[19] S. Ohno, "Products of composition and differentiation between Hardy spaces," Bulletin of the Australian Mathematical Society, vol. 73, no. 2, pp. 235-243, 2006.

[20] S. Stević, "Composition followed by differentiation from $H^{\infty}$ and the Bloch space to $n$th weighted-type spaces on the unit disk," Applied Mathematics and Computation, vol. 216, no. 12, pp. 3450-3458, 2010.

[21] W. Yang, "Products of composition and differentiation operators from $Q_{K}(p, q)$ spaces to Bloch-type spaces," Abstract and Applied Analysis, vol. 2009, Article ID 741920, 14 pages, 2009.

[22] H. Li and X. Fu, "A new characterization of generalized weighted composition operators from the Bloch space into the Zygmund space," Journal of Function Spaces and Applications, vol. 2013, Article ID 925901, 6 pages, 2013.

[23] S. Stević, "Weighted differentiation composition operators from the mixed-norm space to the $n$th weighted-type space on the unit disk," Abstract and Applied Analysis, vol. 2010, Article ID 246287, 15 pages, 2010.

[24] S. Stević, "Weighted differentiation composition operators from $H^{\infty}$ and Bloch spaces to $n$th weighted-type spaces on the unit disk," Applied Mathematics and Computation, vol. 216, no. 12, pp. 3634-3641, 2010.

[25] X. Zhu, "Generalized weighted composition operators on weighted Bergman spaces," Numerical Functional Analysis and Optimization, vol. 30, no. 7-8, pp. 881-893, 2009.

[26] S. Ye and Z. Zhuo, "Weighted composition operators from Hardy to Zygmund type spaces," Abstract and Applied Analysis, vol. 2013, Article ID 365286, 10 pages, 2013.

[27] S. Stević, "Generalized composition operators between mixednorm and some weighted spaces," Numerical Functional Analysis and Optimization, vol. 29, no. 7-8, pp. 959-978, 2008.

[28] S. Ye and J. Gao, "Extended Cesáro operators between different weighted Bloch-type spaces," Acta Mathematica Scientia A, vol. 28, no. 2, pp. 349-358, 2008 (Chinese).

[29] C. C. Cowen and B. D. MacCluer, Composition Operators on Spaces of Analytic Functions, Studies in Advanced Mathematics, CRC Press, Boca Raton, Fla, USA, 1995.

[30] M. Tjani, Compact composition operators on some Moebius invariant Banach spaces [Ph.D. thesis], Michigan State University, 1996.

[31] K. Madigan and A. Matheson, "Compact composition operators on the Bloch space," Transactions of the American Mathematical Society, vol. 347, no. 7, pp. 2679-2687, 1995. 


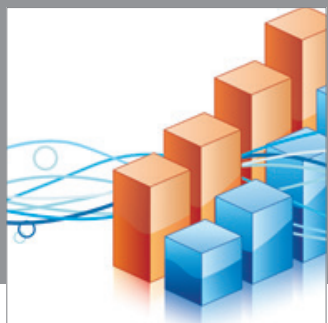

Advances in

Operations Research

mansans

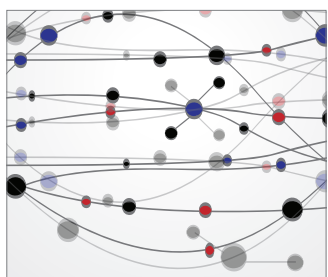

The Scientific World Journal
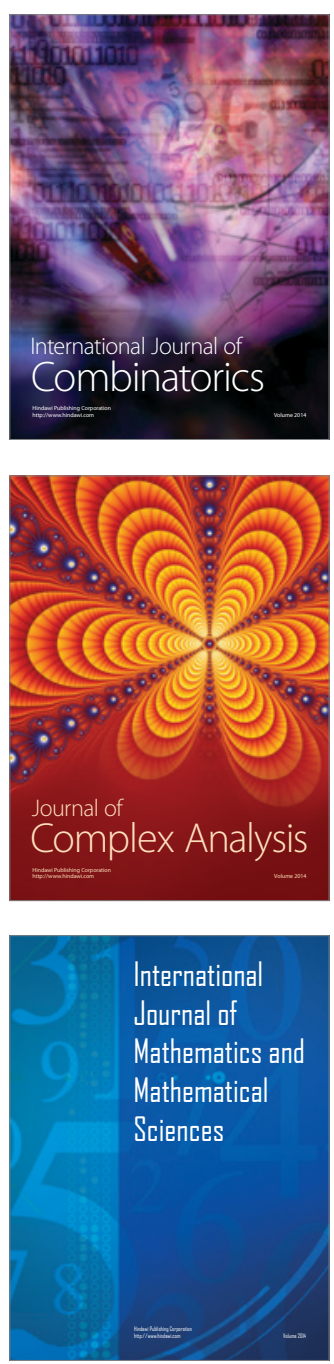
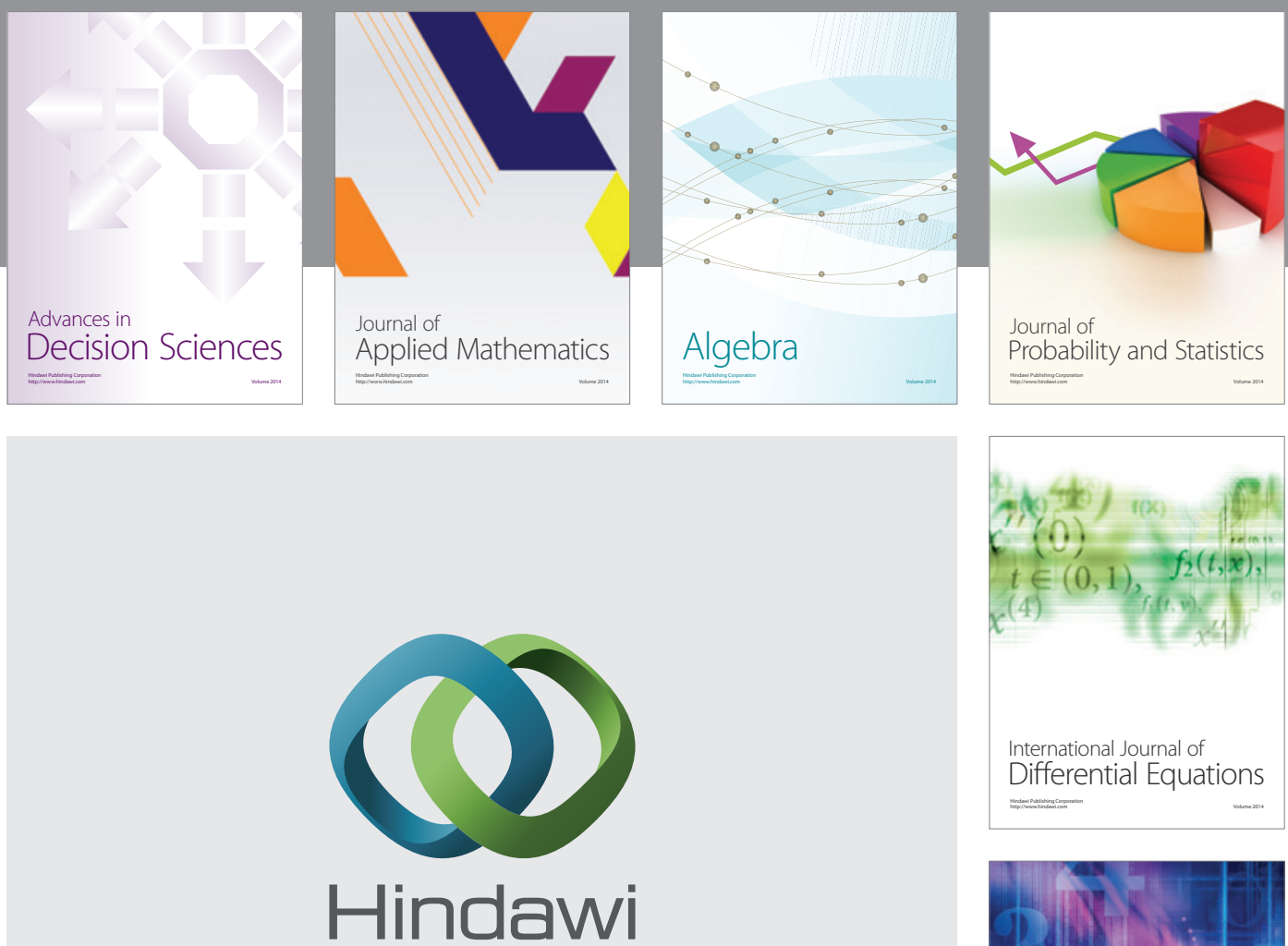

Submit your manuscripts at http://www.hindawi.com
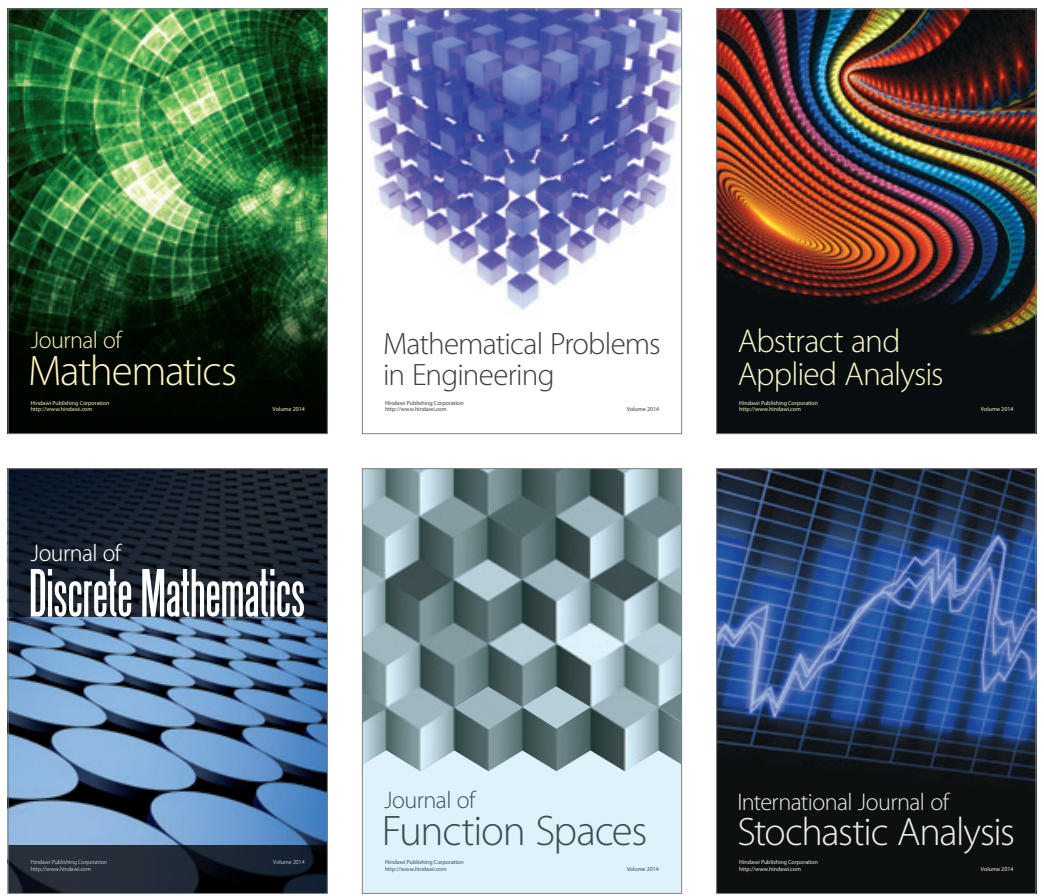

Journal of

Function Spaces

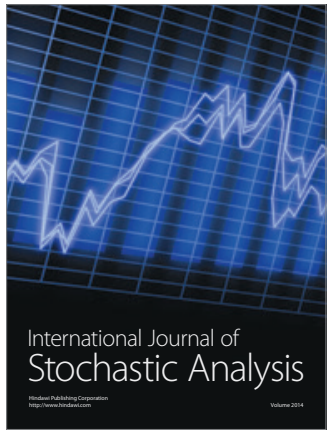

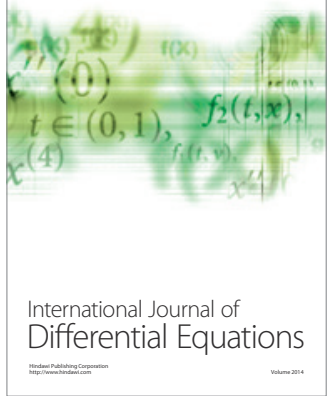
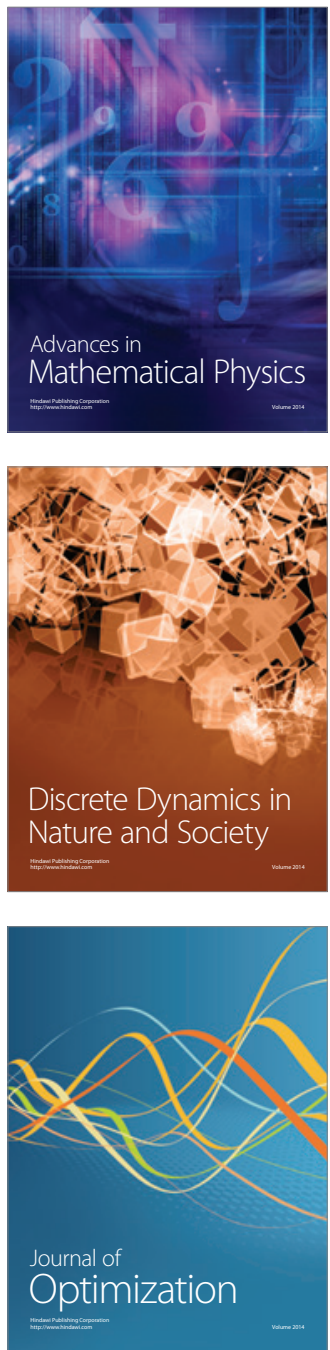